\title{
Propiedad intelectual y evolución de la naturaleza compleja de los vehículos eléctricos*
}

Artemio Chávez** y Arturo Lara***

\section{RESUMEN}

En el marco de la nueva economía institucional y de los sistemas complejos se determina la existencia de la tragedia de los anticomunes y de patentes traslapadas para el caso de los vehículos eléctricos a partir del análisis de las clases tecnológicas de las patentes pertinentes extraídas de la base de datos de la USPTO para el periodo 1976-2012. Usando la teoría de redes, se describe la evolución de la tecnología de los vehículos eléctricos como una red que ha pasado de estar relativamente desconectada a una conectada jerárquicamente en la que: 1) el sistema tecnológico se ordena en subsistemas mediante un proceso simultáneo de integración y "modularización” y 2) se verifica la relación entre la complejidad tecnológica del vehículo eléctrico y la existencia de patentes traslapadas. Metodológicamente, se muestra que analizar clases tecnológicas mediante la teoría de redes es un buen instrumento para el análisis y la determinación del fenómeno de patentes traslapadas.

Palabras clave: tecnologías, tecnología, derechos de propiedad intelectual, patentes, licencias, activos intangibles.

Clasificación JEL: O340.

\begin{abstract}
As part of the new institutional economics and complex systems we determine the existence of the tragedy of the anti-commons and the patent thicket in the case of electric vehicles from the analysis of technological classes drawn from relevant patents database of the USPTO for the period 1976-2012. Using network theory, we describe the evolution of electric vehicle technology, which has grown from a relatively disconnected network to a hierarchically connected network in which 1) the technological system is organized into subsystems using a simultaneous process of integration and modularization and 2) verifying the relationship between the technological complexity of the electric vehicle and the existence of patent thicket. Methodologically we show that analysis of technology classes with network theory is a good tool for the analysis and determination of patent thicket.
\end{abstract}

Keywords: technologies, technology, intellectual property rights, patents, licensing, intangible assets.

JEL classification: $\mathrm{O} 340$.

\footnotetext{
* Fecha de recepción: 10/09/2013. Fecha de aprobación: 16/12/2013.

** Investigador del Programa de Estudios sobre Complejidad, Cognición e Instituciones de la Universidad Autónoma Metropolitana-Xochimilco. Correo electrónico: artemio79@hotmail.com.

*** Profesor-investigador del Programa de Estudios sobre Complejidad, Cognición e Instituciones de la Universidad Autónoma Metropolitana-Xochimilco. Correo electrónico: alararivero35@ gmail.com y alara@correo.xoc.uam.mx.
} 


\section{INTRODUCCIÓN}

En el desarrollo de la teoría institucional de la acción colectiva (Ostrom, 1990 y 2005; Poteete, Janssen y Ostrom, 2011), se encuentran problemas diferentes al de la administración de los recursos tradicionales de uso común (agua, bosques, tierra, etcétera (Heller, 2008); uno de ellos es el relacionado con el conocimiento. ${ }^{1} \mathrm{Si}$ el conocimiento y la propiedad intelectual que se requieren para desarrollar un bien o una tecnología se encuentran fragmentados entre muchos agentes rivales que bloquean su uso u explotación, se dice que existe el fenómeno de patentes traslapadas ( patent thicket) y entonces dicho bien puede ser subutilizado, ${ }^{2}$ conduciendo a la "tragedia de los anticomunes" (Heller, 1998). ${ }^{3}$ De igual modo que el concepto de tragedia de los comunes, el de tragedia de los anticomunes está impulsando trabajo empírico, construcción de modelos formales y refinamiento de la teoría económica institucional. Se ha encontrado evidencia de que la tragedia de los anticomunes se da en la investigación biomédica (Heller y Eisenberg, 1998) y en el desarrollo de la genómica (Van Overwalle, 2009; Osorio y Lara, en prensa). Asimismo, hay estudios que señalan que la existencia de patentes traslapadas tiene el efecto de impedir el proceso de innovación (Shapiro, 2001).

A pesar de que los vehículos eléctricos son bienes complejos constituidos por tecnologías complementarias, no existen estudios sobre este problema en el sector automotriz. Así, el propósito de este artículo es el de reconstruir y explicar la evolución de la naturaleza tecnológica compleja de los vehículos eléctricos -a partir de la base de datos de la United States Patent and Trademark Office (USPTO) para el periodo 1976-2012-4 y la relación que existe entre ésta y los de-

\footnotetext{
${ }^{1}$ Ostrom (1990) establece el "conocimiento" como un elemento fundamental que incide sobre la acción colectiva, porque, por un lado, el "conocimiento común" del problema colectivo permite a los participantes llegar a acuerdos. Y porque, por otro, existen distintos tipos y niveles de información entre los actores que inciden directamente en la configuración de la arena de acción (Ostrom, 2005). Así mismo, el conocimiento puede ser estudiado como un recurso de uso común (Hess y Ostrom, 2003).

${ }^{2}$ Shapiro (2001, p. 120) define la noción de patent thicket (aquí traducida como "patentes traslapadas") así: "[...] a dense web of overlapping intellectual property rights that a company must hack its way through in order to actually commercialize new technology". A su vez, la tragedia de los comunes se expresa no en la subutilización, sino en el agotamiento del recurso (Ostrom, 1990).

${ }^{3}$ Para reconstruir y explicar cómo emerge la forma del dilema social es útil considerar el estudio de la naturaleza del bien, la estructura de la situación de acción y las características de los participantes (Ostrom, 2005).

${ }^{4}$ De la base de datos de la USPTO se obtuvieron 2358 patentes vinculadas con el desarrollo de los vehículos eléctricos. Véase la nota 13.
} 
rechos de propiedad intelectual en posesión de diversos agentes. Las preguntas centrales de este trabajo son las siguientes: ¿de qué manera la teoría de los sistemas complejos permite identificar la existencia y evolución de una densa red de conocimientos tecnológicos, propiedad intelectual y agentes en el desarrollo de los vehículos eléctricos? Y, ¿en qué medida la evolución de la naturaleza del bien explica la existencia de patentes traslapadas y la tragedia de los anticomunes?

En la primera parte del presente estudio se discute la naturaleza del conocimiento y se explica en qué medida su fragmentación y la de la propiedad intelectual asociada pueden generar problemas de subutilización. En un segundo apartado, se describe cómo evoluciona la complejidad de un bien a partir de su arquitectura (modular/integral). En la tercera sección, se describe de forma general la actividad inventiva de los vehículos eléctricos a partir de las patentes pertinentes y se introducen las clases tecnológicas como insumo para describir el sistema. Con base en ello y utilizando la teoría de los sistemas complejos, se explica, en la cuarta parte, cómo la invención tecnológica en los vehículos eléctricos se transformó de una red de conocimiento pequeña y desconectada en una red de conocimiento compleja, extensa y jerárquica. En la quinta sección, se presenta evidencia de que el conocimiento se encuentra fragmentado principalmente en las áreas altamente conectadas, donde se encuentran localizadas empresas rivales. Así, se identifica la presencia de patentes traslapadas en el proceso de invención de los vehículos eléctricos y un escenario de anticomunes.

\section{CONOCIMIENTO, PATENTES TRASLAPADAS Y ANTICOMUNES}

De acuerdo con Hess y Ostrom (2003), en este estudio se considera al conocimiento indistintamente como datos, información o conocimiento propiamente dicho. Es decir, los datos son una parte de la información y el ordenamiento y uso de la información es conocimiento. Si se considera al conocimiento como un recurso, cabe preguntarse de qué tipo de recurso se trata. La respuesta es que, dependiendo de su naturaleza y del contexto institucional, puede ser público, común, de club o privado. ${ }^{5}$

Hay dos aspectos relevantes sobre la naturaleza del conocimiento. El primero consiste en que aunque éste sea un bien intangible, existe en la medida que tiene una base física que lo contiene, por ejemplo, la memoria de una persona, un libro, una base de datos electrónica o una comunidad (Hess y Ostrom, 2003;

\footnotetext{
${ }^{5}$ Véase Ostrom (2005, p. 9, figura 1.1).
} 
Heller, 2008), de tal modo que la forma de esta base determina la imposibilidad o posibilidad y grado de sustracción del bien. ${ }^{6}$ Por otra parte, el arreglo institucional también se encuentra determinado por la naturaleza del bien y esto es especialmente relevante en el caso de los derechos de propiedad intelectual. ${ }^{7}$ Las reglas de propiedad crean formas artificiales de exclusión (Coriat, 2011). Así, por ejemplo, el conocimiento de una tecnología puede ser accesible mediante una base de datos de patentes y relativamente fácil de reproducir, pero imposible de desarrollar o explotarse (por lo menos legalmente) si el propietario de ese conocimiento se niega a otorgar la licencia correspondiente. ${ }^{8}$

Estos dos aspectos iluminan dimensiones clave de la evolución de la capacidad inventiva en el sector de los vehículos eléctricos. En la innovación de un producto complejo, como lo es el vehículo eléctrico, ninguna persona o empresa puede desarrollar el producto de manera individual, por el contrario, se requiere de una población numerosa de agentes altamente especializados.

Debido a lo anterior, en la medida en que el conocimiento y la propiedad intelectual asociada requeridos para el desarrollo de una tecnología compleja se encuentren fragmentados en distintas patentes complementarias, cualquier agente tiene la capacidad de negarse a dar su licencia (problema de retención) y, por ende, la posibilidad de bloquear la innovación o incrementar sus costos. El resultado es inevitable: la subutilización del bien o la tragedia de los anticomunes (Heller, 2008).

En el centro de este último problema se encuentra una red de propietarios de patentes complementarias traslapadas, la cual puede estar originada por tres factores: la complejidad del bien (IPO, 2011), la estrategia de algún participante (Bessen, Ford y Meurer, 2012) $)^{9} \mathrm{o}$ los problemas burocráticos surgidos en

\footnotetext{
${ }^{6}$ Es el caso de una biblioteca (recurso), en la que el uso de un libro por parte de una persona impide que otra acceda a él simultáneamente (sustracción).

${ }^{7}$ Por ejemplo, una invención tecnológica, que es conocimiento, es relativamente sencilla de proteger legalmente por medio de los derechos de propiedad intelectual, mientras que una lengua, que también es conocimiento, es propio de un régimen de dominio público.

${ }^{8} \mathrm{El}$ avance tecnológico digital ha hecho que los receptáculos del conocimiento sean cada vez más económicos y fáciles de reproducir, de tal modo que tanto la rivalidad como la posibilidad de exclusión se reducen.

${ }^{9}$ Existen dos posibles casos en los que la presencia de patentes traslapadas tiene un origen estratégico: a) cuando una empresa o inventor, independientemente de su tamaño o participación en el mercado, bloquea con patentes un área tecnológica en la que quiere innovar (IPO, 2011) y b) cuando un tipo de entidad no practicante (patent troll), es decir, que no se dedica a la producción, adquiere patentes para obtener beneficios posteriores con su licenciamiento (Bessen, Ford y Meurer, 2012).
} 
las distintas oficinas de patentes (Bessen y Maurer, 2008).${ }^{10}$ El presente estudio, se limita exclusivamente a la primera causa. ${ }^{11}$ Para ello, se buscará inicialmente responder la siguiente pregunta: ¿Cómo representar y analizar la evolución de la arquitectura de un sistema tecnológico complejo como es el de los vehículos eléctricos?

\section{COMPLEJIDAD, SISTEMA INTEGRAL Y SISTEMA MODULAR}

En la teoría de la innovación tecnológica se describen dos arquitecturas que representan, desde el punto de vista espacial y funcional, la manera en que los componentes y las funciones interactúan. En la industria, y en el sector automotriz en particular, se han identificado principalmente las siguientes: el sistema integral y el modular (Ulrich y Eppinger, 2009). En el primero todos los elementos están estrechamente vinculados con el resto. Por su parte, la arquitectura modular se define de la siguiente manera: "modules are units in a larger system that are structurally independent of one other, but work together. The system as a whole must therefore provide a framework -an architecture-that allows for both independence of structure and integration of function" (Baldwin y Clark, 1999, p. 63). Así, el sistema modular se divide en subsistemas o módulos, los cuales guardan una relativa autonomía entre ellos, en tanto que al interior de cada módulo sus componentes interactúan de manera estrecha.

¿Cómo representar formalmente la complejidad de estos dos sistemas? ${ }^{12}$ La teoría de los sistemas complejos brinda distintas posibilidades (Kauffman,

\footnotetext{
${ }^{10}$ Las oficinas que las registran se enfrentan a diversos problemas que afectan la calidad de la patente: un alto número de solicitudes por revisar, cambios en las tecnologías para los cuales es difícil encontrar examinadores expertos, lenguajes y codificaciones novedosas que dificultan la asignación precisa de claims, y un estado del arte previo cada vez más amplio que es prácticamente imposible de rastrear en su totalidad (Bessen y Maurer, 2008).

${ }^{11}$ El estudio de los otros dos factores requiere un tratamiento separado. Se trata de problemas que demandan un espacio y atención que escapan al presente análisis. Sin embargo, es importante señalar que la literatura y debate sobre el fenómeno de las patentes traslapadas se ha incrementado en la última década tanto en lo que toca a su precisión conceptual, como en sus múltiples causas, las formas de medirlo y los diversos efectos que produce (Heller y Eisenberg, 1998; Shapiro 2001; Clarkson y DeKorte, 2006; Bessen y Maurer, 2008; Ballardini, 2009; Von Gravenitz, Warner y Harhoff, 2011).

${ }^{12}$ Así mismo, la otra posibilidad se relaciona con la teoría económica de la innovación especializada en el análisis de los sistemas integrales y modulares (Baldwin y Clark, 1999; Sanchez y Mahoney, 2003). Por razones analíticas y de mayor riqueza conceptual e instrumental, este trabajo se inclina por la teoría de los sistemas complejos.
} 
1993; Holland, 1996; Mandelbrot, 1997; Watts, 1999). De acuerdo con Kauffman (1993) y Frenken, Marengo y Valente (1999), cualquier sistema se compone básicamente de un conjunto de partes $(N)$ y de sus relaciones $(k)$. Así, la complejidad del sistema se puede medir a partir de ambas dimensiones. Tiene mayor significado, sin embargo, la dimensión $k$, toda vez que refleja las posibles combinaciones de $N .{ }^{13}$

Independientemente del número de componentes, en un sistema integral, la cantidad de relaciones entre ellos (el valor de $k$ ) es el máximo posible. Esto es, cada uno de los componentes del sistema se vincula con todos los demás componentes. Formalmente $k=N(N-1) / 2$. En el caso del sistema modular, las relaciones de los componentes al interior de cada módulo son mayores, en tanto que las relaciones entre módulos son menores.

De este modo, en un sistema integral: 1) Pequeños cambios en un parámetro de diseño pueden desencadenar múltiples ajustes a nivel del sistema. 2) Lo anterior trae como consecuencia que tanto los costos de coordinación como de implementación de la innovación sean altos. 3) Por ello, la velocidad del cambio a nivel de componentes es relativamente más lenta que en el sistema modular. 4) Si bien el espacio de posibilidades de innovación del sistema en su conjunto es mayor y existe la probabilidad de encontrar un máximo global, se requiere de una serie de condiciones adicionales, en particular condiciones organizacionales excepcionales. ${ }^{14}$ (Baldwin y Clark, 1999; Ulrich y Eppinger, 2009; Sanchez y Mahoney, 2003; Lara y García, 2005).

En tanto, en el sistema modular: 1) El cambio en un parámetro de diseño sólo implica ajuste en un módulo, dejando inalterado el resto del sistema. 2) Lo anterior permite reducir los costos del cambio y el tiempo de desarrollo. 3) También aumenta la flexibilidad y la diversidad de productos (diferenciación). 4) Al reducir las posibilidades de exploración, cabe la posibilidad de elegir un diseño

\footnotetext{
${ }^{13}$ Por ejemplo, un sistema de poleas es en realidad un sistema bastante simple. Una polea adicional, potencia de forma exponencial la reducción de la fuerza necesaria para mover un objeto, pero no vuelve más complejo al sistema. Así, si se tuviera un sistema de diez mil poleas se podría decir que aumentó su grado de complejidad, sin embargo, difícilmente se podría afirmar que lo hizo de forma significativa. Por otro lado, un sistema como un vehículo automotor requiere de muchos componentes (cerca de diez mil piezas) (Ulrich y Eppinger, 2009) que se relacionan de múltiples formas y en diferentes grados.

${ }^{14}$ En el caso de un sistema tecnológico complejo como un vehículo eléctrico, se requiere que todas las organizaciones y agentes converjan para acordar de manera conjunta una nueva arquitectura del producto, lo cual resulta inviable dado que las capacidades y sus portadores están distribuidos de manera heterogénea en distintos espacios geográficos y múltiples campos tecnológicos.
} 
subóptimo, es decir, un óptimo local (Baldwin y Clark, 1999; Sanchez y Mahoney, 2003; Lara y García, 2005; Ulrich y Eppinger, 2009).

En los estudios sobre la innovación tecnológica, el debate académico se ha centrado en si existen o no posibilidades de que el sector automotriz transite del sistema integral al sistema modular (Fixon y Sako, 2001; Langlois, 2002; Pandremenos, Paralikas, Salonitis y Chryssolouris, 2009). Dicha discusión, sin embargo, se presenta de forma dicotómica. Es decir, no se considera que el cambio total y radical de un sistema a otro resulte improbable y riesgoso, ni que los procesos de desacoplamiento y agrupación de los componentes ("modularización") y el incremento en la complejidad al interior de un subsistema sean graduales. A continuación, se propone un esquema explicativo de transición del sistema integral al modular desde esta perspectiva.

\section{Vinculación compleja de los sistemas integrales y modulares}

Inicialmente, un sistema tiene un nivel de complejidad relativamente bajo ( $N$ y $k$ bajas), pero al enfrentar nuevos problemas, se añaden nuevos componentes y relaciones. Por ejemplo, el desarrollo del vehículo eléctrico empieza con el uso de la batería tradicional LSI de plomo-ácido (Lara y Salazar, 2013). Sin embargo, dado que este dispositivo no acumula suficiente energía, se buscaron nuevos materiales activos, como el níquel-metal hidruro, el níquel-cadmio, el litio-ion, entre otros (Lara y García, 2005; Reyes, 2012). Así, se desarrolla una nueva generación de baterías avanzadas que exigen el diseño y la integración de subsistemas de seguridad y medición actualizados. Estos nuevos diseños expanden el número de componentes y de sus relaciones (de forma que se incrementan $N$ y $k$ ). Pero lo más relevante de este proceso es que la evolución del vehículo eléctrico conduce a que una parte del sistema se desacople y emerja un nuevo subsistema como el de la batería (Lara, en prensa). Este nuevo subsistema o módulo desacoplado especializado desarrolla asimismo nuevas formas de interacción en su interior como lo hace un sistema de tipo integral o de tipo modular. Todos estos cambios en la naturaleza del producto también redefinen de formas más imbricadas las relaciones entre los distintos agentes. Esta perspectiva analítica se representa en el cuadro 1.

Así, si esta perspectiva analítica representa mejor la realidad, el debate del sistema integral versus el sistema modular debe ser replanteado. Más adelante se examina, a partir de la información contenida en las patentes, la actividad inventiva en el desarrollo de los vehículos eléctricos. ¿Es posible identificar 
empíricamente en los vehículos eléctricos la existencia de procesos evolutivos como se sintetiza en el cuadro 1 ? Creemos que sí.

\section{Cuadro 1. Relación de la evolución de las complejidades de los SI y los SM}

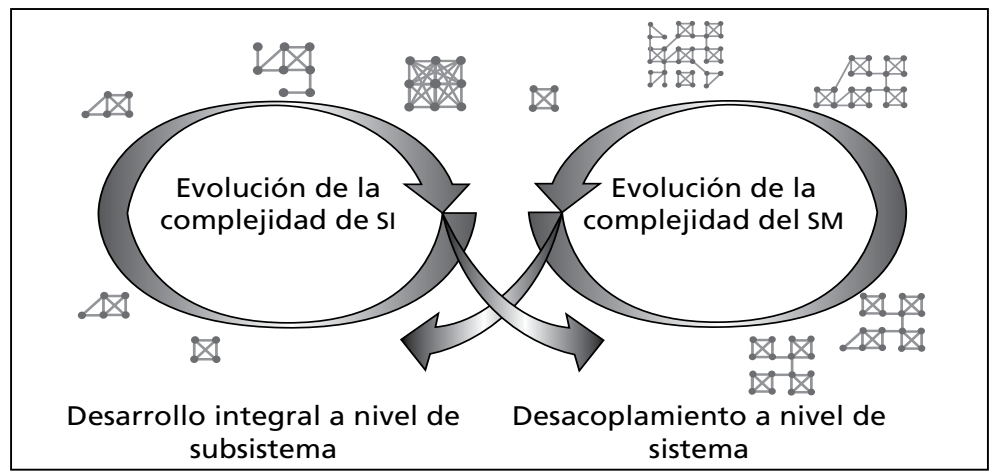

Fuente: Elaboración propia. SI: sistema integral, SM: sistema modular.

Dada la naturaleza compleja de lo que se busca describir se ha optado por el uso del análisis de redes, con base en la información que se extrajo de la USPTO. ${ }^{15}$ El análisis de redes permite describir con precisión la densidad en un área de estudio, la dimensión de la estructura completa del sistema y la formación emergente de distintos grupos (subsistemas o módulos) a partir de su cercanía relativa. Esta herramienta es útil para representar la evolución de la complejidad de los vehículos eléctricos. Éste es el objeto de las siguientes secciones.

\footnotetext{
${ }^{15}$ Para este estudio se consideraron las patentes de la USPTO por dos razones: a) la base de datos de Estados Unidos es confiable y accesible y b) el mercado estadounidense es uno de los más importantes del mundo, donde se espera que las distintas empresas automotrices busquen proteger su conocimiento. La base de datos se construyó por medio de la búsqueda de patentes que pertenecen a las clases 180/65.1, 180/65.21 y 701/22. Estas clases se escogieron porque la propia USPTO (s.f.) considera que los vehículos eléctricos pertenecen a dichas clases. La base de datos cuenta con 2358 patentes que van desde el 1 de enero de 1976 hasta el 20 de noviembre de 2012. Todas las patentes están clasificadas según el área de conocimiento técnico al que pertenecen. Cada oficina de patentes tiene su propia clasificación y una adicional que pertenece a la Organización Mundial de la Propiedad Intelectual (WIPO, por sus siglas en ingles). Así, cuando se hace referencia a la clase se indica con ello el dominio tecnológico al que pertenece una patente. Es importante señalar que estas patentes incluyen a todos los vehículos eléctricos, es decir, no sólo a los de la industria automotriz, sino también a los terapéuticos, como sillas de ruedas eléctricas, o vehículos eléctricos montables para niños. Estos medios de transporte no son retirados del análisis por dos razones: 1) se sesgaría la base con un criterio diferente y subjetivo respecto al que se usó para construirla y 2) se eliminaría la posibilidad de observar relaciones entre este tipo de productos y los propios de la industria automotriz.
} 


\section{ACTIVIDAD INVENTIVA EN LOS VEHÍCULOS ELÉCTRICOS}

Los vehículos eléctricos tienen una historia que se inicia a finales del siglo XIX, sin embargo, la superioridad de los vehículos de combustión interna impidió el desarrollo de aquéllos (Cowan y Hultén, 1996; Lara y Salazar, 2013). Frente a los cambios en los precios de los combustibles fósiles desde la década de los setenta y el incremento del rigor en las normas institucionales para reducir las emisiones contaminantes de los vehículos, principalmente en los ochenta y los noventa, la industria automotriz se vio en la necesidad de crear nuevos transportes que sustituyeran al motor de combustión interna (National Research Council, 2013; Lara y Salazar, 2013). De las alternativas que ha encontrado la industria, el diseño de los vehículos eléctricos parece ser una de las más importantes tanto por la actividad inventiva que ha desarrollado como por los incentivos que los gobiernos de Estados Unidos, Europa y Japón (que son las regiones de origen de los principales fabricantes de vehículos) han implementado (Juliussen y Robinson, 2010; Lara y Salazar, 2013). Así, al inicio de la década de los noventa, la actividad de invención, reflejada en el número de patentes, se incrementó exponencialmente, pasando de 11 patentes en 1990 a 389 en 2012 (gráfica 1) y de seis distintas empresas o alianzas empresariales que patentaron en 1990 a 97 en 2012 (gráfica 2).

Gráfica 1. Número de patentes para vehículos eléctricos, 1976-2012

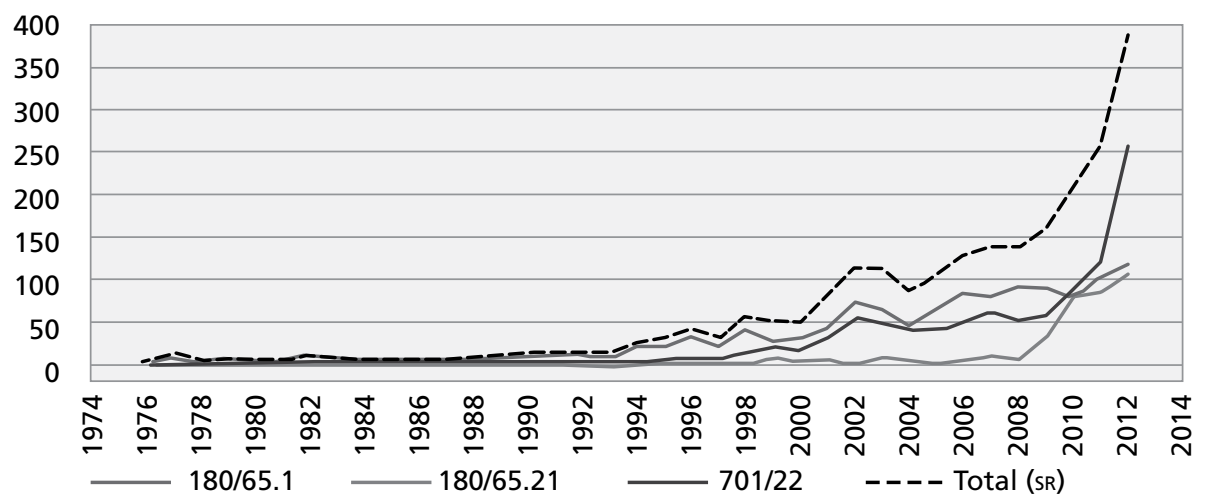

SR: $\sin$ repeticiones.

Fuente: Elaboración propia con información obtenida de la USPTO. Base de datos UAM/PECCI. Proyecto "Sistemas Complejos Adaptables y Cooperación Tecnológica", CONACYT No. I0017156204.

A nivel agregado, durante veinte años, la evolución de la actividad inventiva se concentró en la clase 180/65.1 (Motor vehicles; electric power). Sin 
embargo, desde 2008 y hasta 2012, las clases 701/22 (Data processing: vehicles, navigation, and relative location; electric vehicle) y 180/65.21 (Motor vehicles; electric power, hybrid vehicle) se han convertido en las áreas de mayor desarrollo (gráfica 1). Esto indica que el centro de la actividad de invención transita del diseño de dispositivos tangibles para vehículos eléctricos e híbridos al procesamiento de datos, lo que se debe al incremento de las unidades de control electrónico ${ }^{16}$ (Juliussen y Robinson, 2010), fenómeno que también se da en los transportes tradicionales (Lara, 2014).

\section{Gráfica 2. Número de agentes con patentes para vehículos eléctricos, 1976-2012}

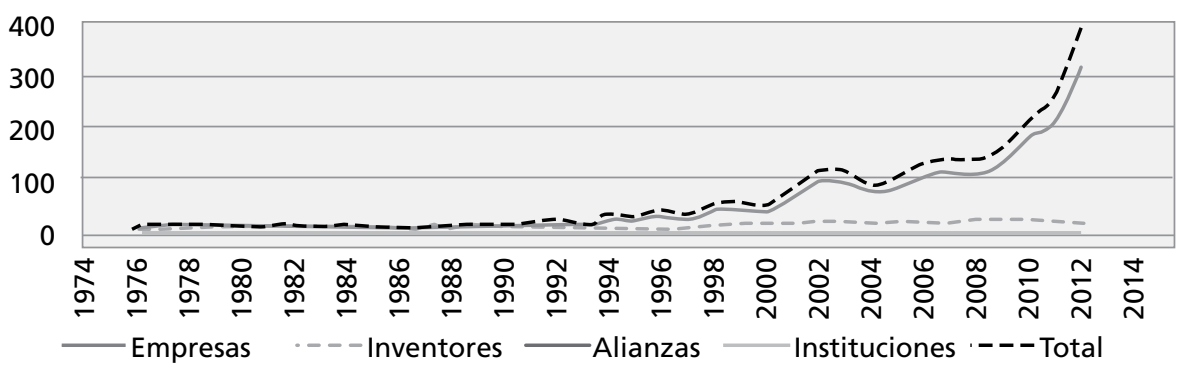

VE: vehículos eléctricos. El total de inventores individuales se considera como un solo agente.

Fuente: Elaboración propia con información obtenida de la USPTO. Base de datos UAM/PECCI. Proyecto "Sistemas Complejos Adaptables y Cooperación Tecnológica", CONACYT No. 10017156204.

Las clases tecnológicas pueden interpretarse como el espacio de exploración o de investigación de los agentes. ${ }^{17}$ En 1976, las patentes vinculadas a los vehículos eléctricos sólo contaban con ocho clases; en 2012 el total acumulado de clases ascendió a 2 394. Por lo tanto, la actividad inventiva aumentó no sólo en volumen, sino, sobre todo, en nuevas áreas de exploración de conocimientos $\mathrm{y}$, por ende, en complejidad. Se podría considerar que el crecimiento en el volumen de clases está asociado al crecimiento en el número de patentes, pero sería parcialmente cierto, ya que explica sólo una parte del panorama. Al ponderarse

\footnotetext{
${ }^{16} \mathrm{ECU}$, por sus siglas en inglés.

${ }^{17}$ Todas las patentes se clasifican según los manuales de cada oficina de patentes. La clasificación hace referencia a un área de conocimiento, por ejemplo, la clase 701/22 de la USPTO se refiere al procesamiento de datos de los vehículos eléctricos, mientras que la clase 180/65.1 al motor eléctrico. No existe límite en el número de clases que puede contener una patente y entre más tenga puede interpretarse como un conocimiento más especializado.
} 
las clases por año, se observa un patrón en el que, en promedio, hay unos periodos cuando las clases aumentan y otros en los que se reducen (gráfica 3).

Gráfica 3. Promedio de clases para vehículos eléctricos, 1976-2012

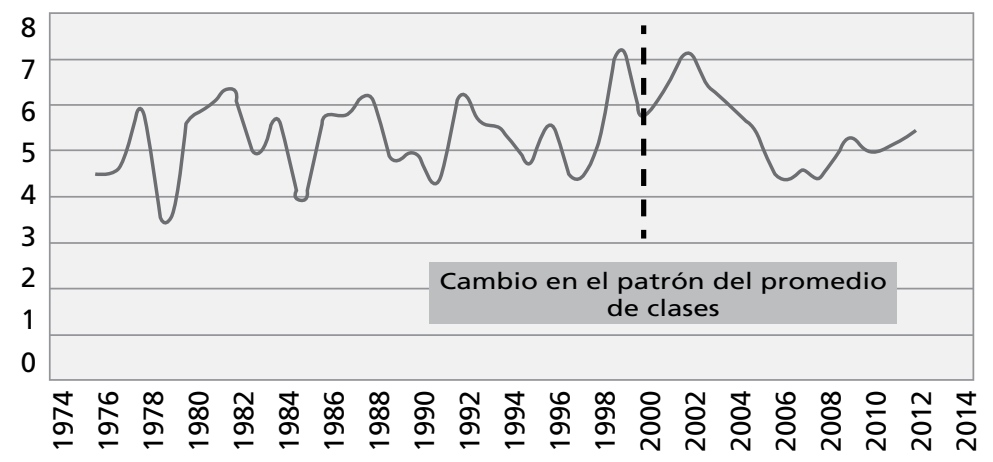

Fuente: Elaboración propia con información obtenida de la USPTO. Base de datos UAM/PECCI. Proyecto "Sistemas Complejos Adaptables y Cooperación Tecnológica", CONACYT No. 10017156204

Es probable que este patrón se deba al hecho de que cuando los agentes enfrentan problemas particulares, exploran nuevas soluciones, lo que se expresa en un mayor número promedio de clases. Esto es consecuente con el aumento de la complejidad debido la incremento de nuevos componentes $(N)$ y sus posibles relaciones $(k)$ propio de un desarrollo integral. Las nuevas soluciones plantean, a su vez, nuevos problemas que exigen soluciones cada vez más especializadas, lo cual se expresa en la reducción en el número promedio de clases, que en combinación con la cantidad creciente de patentes, implica un aumento en el número de relaciones $(k)$ relativamente mayor al de clases $(N)$. De esta manera, emerge un proceso cíclico de expansión-contracción del promedio de clases como resultado de la exploración y la especialización crecientes del conocimiento tecnológico, coherente con el proceso que sintetiza la relación entre las arquitecturas integral y modular descrita en la sección anterior.

\section{ANÁLISIS DE CLASES DE LOS VEHÍCULOS ELÉCTRICOS POR MEDIO DE REDES}

Para representar este fenómeno con mayor detalle, se efectúa un análisis por medio de redes, una técnica propia del estudio de los sistemas complejos que permite 
determinar con mayor precisión la evolución de la actividad de invención. ${ }^{18}$ A continuación, se identifican los micropatrones en la evolución de las clases de los vehículos eléctricos.

\section{Micropatrones simples de evolución de las clases tecnológicas}

Los examinadores de la USPTO clasifican las patentes según el área de conocimiento. Una patente puede pertenecer a una sola o a muchas áreas de conocimiento. ${ }^{19}$ Así, es posible vincular una patente con otra a partir de las clases a las que pertenecen. ${ }^{20}$ En la gráfica 4 se presentan las patentes de vehículos eléctricos registradas en $1976:^{21}$ la 3986095 y la 3984742 , que cuentan con cinco y cuatro clases, respectivamente.

El primer micropatrón que se puede observar es el del agrupamiento de clases..$^{22}$ Ambas patentes tienen en común la clase 180/65.1 y es por ello que pueden vincularse. Sin embargo, para una de ellas esta clase representa $16.66 \%$ del total de sus vínculos, mientras que para la otra representa sólo $10 \% .{ }^{23} \mathrm{Como}$ el peso de la clase 180/65.1 es mayor en la patente 3984742 que en la 3986095 , se agrupa con la primera.

${ }^{18}$ Para quien esté interesado en reproducir los resultados o generar nuevos análisis, en el anexo metodológico se presentan los pasos generales que se siguieron para la construcción de las redes incluidas en el presente análisis.

${ }^{19}$ No existe un límite de clases (véase la nota 13).

${ }^{20}$ Esto es, la patente 1 puede tener las clases A, B y C. La patente 2 las clases C, D y E. De tal modo que ambas patentes están conectadas por la clase $\mathrm{C}$ y la red resultante se constituye por cinco nodos (A, B, C, D y E) con 6 vínculos ( $\mathrm{AB}, \mathrm{AC}, \mathrm{BC}, \mathrm{CD}, \mathrm{AB}, \mathrm{CE}$ y $\mathrm{DE})$.

${ }^{21}$ Este año sólo se registraron estas dos patentes.

${ }^{22}$ Este agrupamiento resulta de aplicar el algoritmo Clauset-Newman-Moore en el programa NodexL, el cual agrupa nodos a partir de la optimización de la "modularidad" (Clauset, Newman y Moore., 2004) con el objetivo de encontrar la estructura de la red.

${ }^{23}$ Los vínculos totales de una patente $(k)$ se calculan como $N(N-1) / 2$, donde $N$ es el número de clases de la patente. Así, por ejemplo, en el caso de 1976 para los vehículos eléctricos, la patente 3984742 tiene cuatro clases, de tal modo que el total de vínculos es de seis; cada clase representa, por lo tanto $1 / 6=16.66 \%$ del total. Mientras tanto, la patente 3986095 que cuenta con cinco clases, tiene diez vínculos y, por lo tanto, cada clase representa $10 \%$ del total de sus vínculos. Como la clase 180/65.1 se encuentra en ambas patentes, el algoritmo maximiza el peso relativo de este nodo de tal modo que asocia esta clase (nodo) con el grupo en el que tiene mayor peso. Es por ello, que 1) ambas patentes están conectadas por la clase 180/65.1 y 2) al tener mayor peso relativo esta clase en el grupo de clases que componen la patente 3984742 (16.66\%) es que se agrupa con éstas (líneas obscuras) y no con el grupo de clases que contiene la patente 3986095 (líneas claras) en el que sólo representa $10 \%$. 


\section{Gráfica 4. Red de clases de vehículos eléctricos para 1976. Agrupamiento}

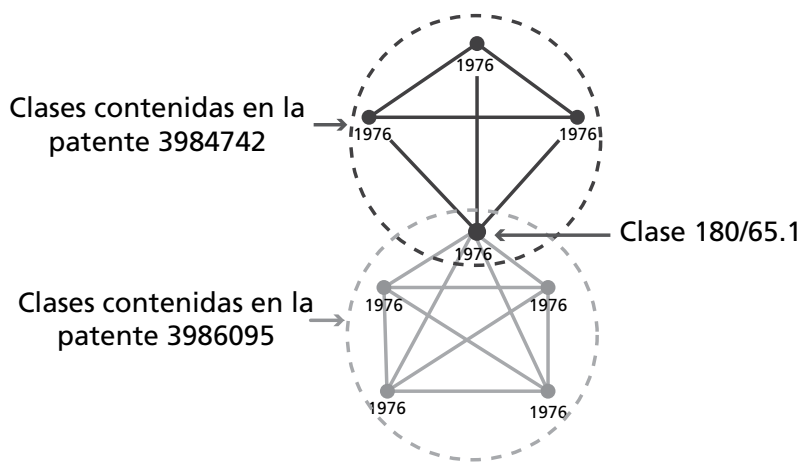

Fuente: Elaboración propia con información obtenida de la USPTO. Base de datos UAM/PECCI. Proyecto "Sistemas Complejos Adaptables y Cooperación Tecnológica", CONACYT No. 10017156204.

Gráfica 5. Red de clases de vehículos eléctricos para 1976-1978.

Derivación, autonomía relativa y combinación

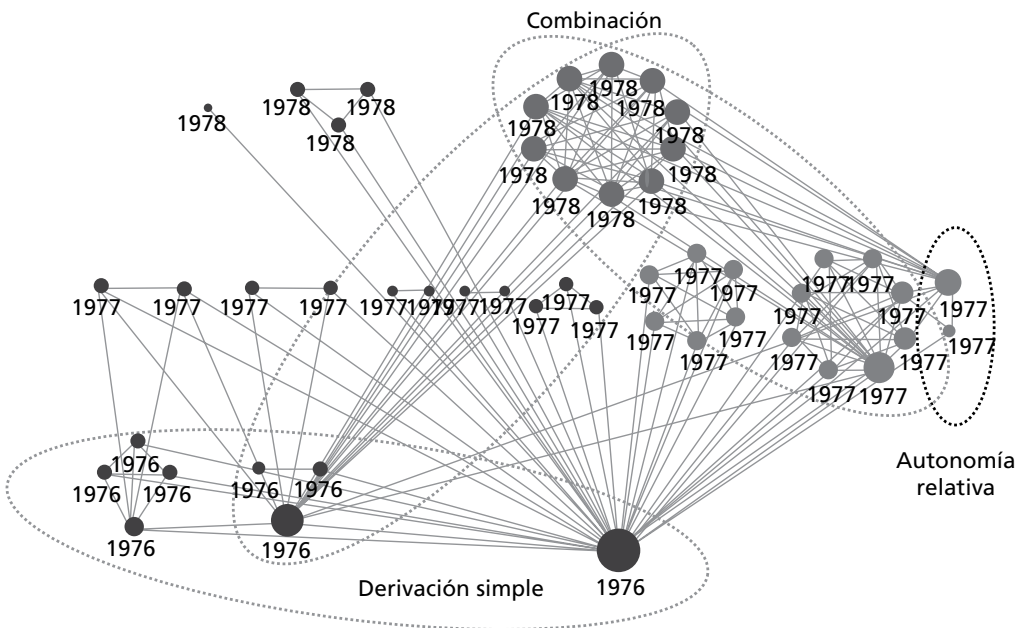

Fuente: Elaboración propia con información obtenida de la USPTO. Base de datos UAM/PECCI. Proyecto "Sistemas Complejos Adaptables y Cooperación Tecnológica", CONACYT No. 10017156204.

En un segundo caso (gráfica 5), y para el periodo 1976-1978, se pueden identificar tres micropatrones ${ }^{24}$ a los que denominamos derivación simple, auto-

${ }^{24}$ Estos micropatrones son resultado de la aplicación del mismo algoritmo Clauset-NewmanMoore para la identificación de estructura en la red. 
nomía relativa y combinación. La derivación simple se presenta cuando hay un conjunto de clases que comparten una clase en común que tiene un mayor grado de conectividad ${ }^{25} \mathrm{y}$ además pertenecen al mismo grupo; la autonomía relativa se presenta cuando hay un conjunto de clases que pertenecen a un mismo grupo, pero en su interior las conexiones son más intensas en algunas clases que en otras, y la combinación se presenta cuando hay un conjunto de clases que pertenecen a un grupo y comparten clases de otros grupos.

\section{Gráfica 6. Red de clases de vehículos eléctricos para 1978. Clases no relacionadas}

Clase 180/65.1 (Motor vehicles; electric power)
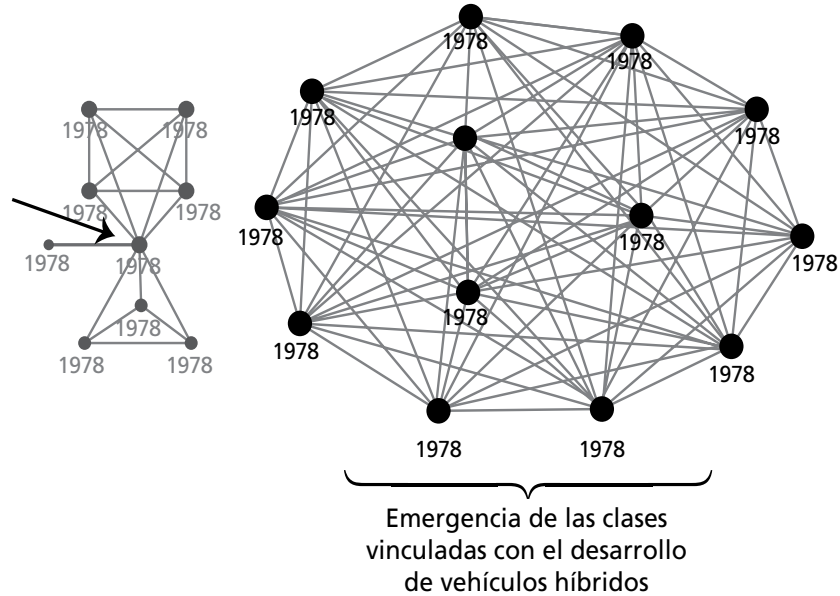

Fuente: Elaboración propia con información obtenida de la USPTO. Base de datos UAM/PECCI. Proyecto "Sistemas Complejos Adaptables y Cooperación Tecnológica", CONACYT No. 10017156204.

Resulta útil representar los fenómenos que emergen en un periodo corto, por ejemplo, el año de 1978 (gráfica 6). En ese lapso emerge un micropatrón: dos agrupamientos de clases no relacionadas. Esto quiere decir que, para diseñar vehículos eléctricos, los agentes exploran nuevas clases (áreas de conocimiento) relativamente ajenas, creándose de esta manera un sistema más complejo. En particular, en dicho año sólo se registraron cuatro patentes (4124086, 4123740, 4094377 y 4090577 ) con 22 clases en total. Tres de estas patentes (la 4124086, la 4123740 y la 4094377) se agrupan alrededor de la clase 180/65.1, con un total de nueve clases, y en el otro grupo se encuentra la patente 4090577, que agrupó

${ }^{25}$ El grado de conectividad es el número de vínculos o enlaces que tiene un nodo. En nuestro análisis de clases, por ejemplo, si una clase tiene 12 vínculos se dice que el grado de conectividad de esa clase es de 12 . 
a 13 clases, todas ellas vinculadas con el desarrollo de vehículos híbridos. Es decir, es un periodo en el que la desconexión implica la exploración de nuevas tecnologías. ${ }^{26}$

\section{Gráfica 7. Red de clases de vehículos eléctricos para 1976-1982. Reagrupamiento}

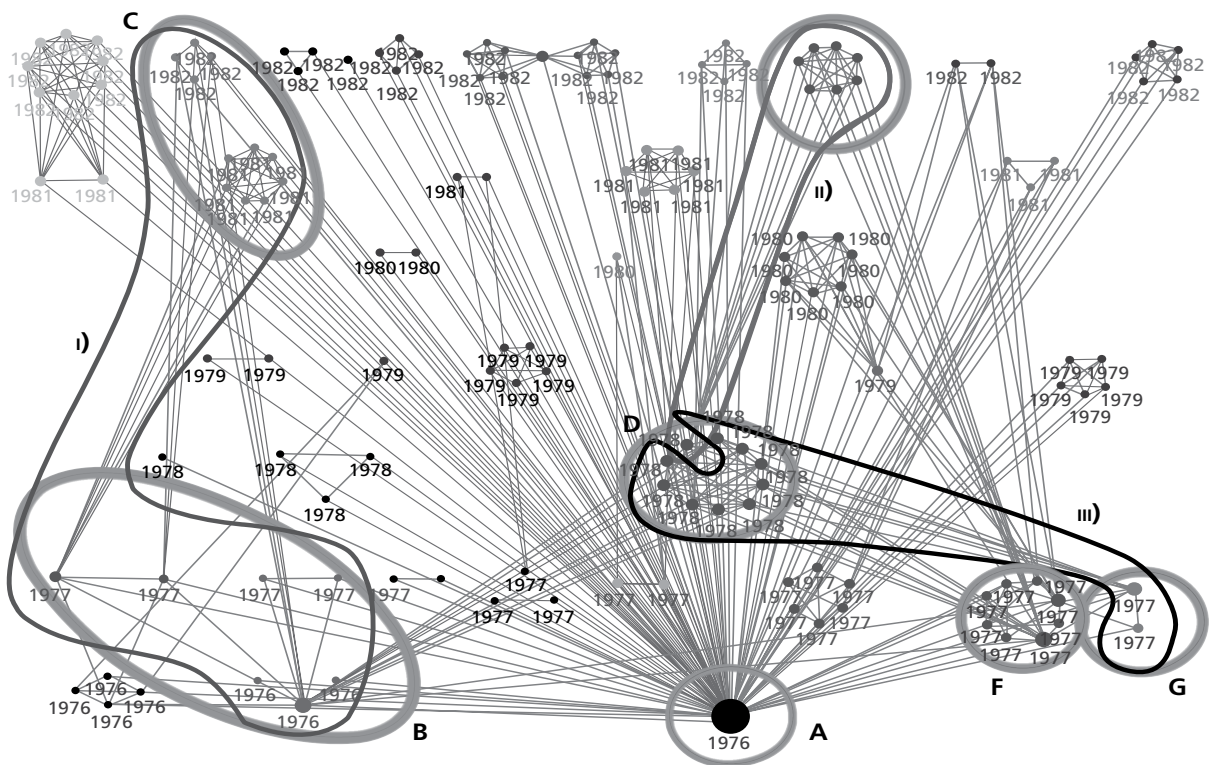

Fuente: Elaboración propia con información obtenida de la USPTO. Base de datos UAM/PECCI. Proyecto "Sistemas Complejos Adaptables y Cooperación Tecnológica", CONACYT No. 10017156204.

Finalmente, otro micropatrón identificable es el de reagrupamiento. Se produce cuando el surgimiento de nuevas clases provoca que las previas cambien de grupo. Este fenómeno puede ser el resultado de distintas trayectorias evolutivas. Por ejemplo: 1) clases que se derivaron de una previa se reagrupan con otras, ${ }^{27}$ 2) clases fuertemente conectadas con un grupo cambian de grupo, ${ }^{28}$

${ }^{26}$ Esto quiere decir que un bajo número de patentes no implica necesariamente poca actividad inventiva, ya que ésta se asocia más con el volumen de clases que con la cantidad de patentes.

${ }^{27}$ En la gráfica 7 se observa que del área A se derivaron de forma simple las clases del área $\mathrm{B}$ (véase la gráfica 5), pero ante la emergencia de las clases del área $\mathrm{C}$, las de $\mathrm{B}$ se reagruparon con estas últimas por su mayor conectividad.

${ }^{28}$ La gráfica 7 muestra que de las clases contenidas en el área D, antes fuertemente vinculadas en un grupo (véase la gráfica 5), se desprende una clase que por su mayor conectividad con las clases del área $\mathrm{E}$ se reagrupa con éstas. 
3) clases que pertenecían a un grupo con autonomía relativa, cambian a otro grupo manteniendo dicha autonomía relativa, ${ }^{29}$ etcétera. Todas estas posibilidades se muestran en la red de clases de vehículos eléctricos correspondiente al periodo 1976-1982 (gráfica 7).

\section{Macroproceso complejo de evolución del sistema tecnológico}

La evolución de los micropatrones incide sobre la estructura del sistema en su conjunto y nos interesa determinar si ésta presenta un proceso de integración y "modularización" como el descrito en la sección II. Para ello, primero se representará gráficamente, y por periodos, la evolución del sistema y posteriormente se analizará éste de forma cuantitativa.

\section{a) Representación gráfica de la evolución de sistema tecnológico}

Para ilustrar gráficamente la evolución del sistema tecnológico de los vehículos eléctricos se ha optado por dividir el periodo en tres fases. La primera fase comprenderá de 1976 a 1995; la segunda, el periodo intermedio de 1984 a 2003, y la tercera, de 1993 a $2012 .{ }^{30}$

Primera fase (1976-1995). En esta primera fase, la clase 180/65.1 domina la conectividad del conjunto del sistema (gráfica 8), en tanto que al interior los micropatrones revelan la creación de subsistemas o subgrupos relativamente más conectados que muestran indicios de separación respecto del subsistema central. Éste es el caso de tres clases que pertenecen a un mismo grupo, pero que mantienen autonomía relativa determinada por su grado de conectividad específica:

${ }^{29}$ Es posible observar en la gráfica 7 que las clases contenidas en el área G y que tenían una autonomía relativa respecto a las clases del grupo F (véase la gráfica 5) se reagrupan con las clases del área D manteniendo dicha autonomía relativa. Este reagrupamiento se debe no sólo al incremento en la conectividad de las clases de estas dos áreas (D y G), sino además porque los pesos relativos de las clases contenidas en las áreas F y D cambiaron por la emergencia de clases que no se vinculan con las clases del área G.

${ }^{30}$ Esta periodización se eligió por las siguientes razones: 1) El tamaño de cada lapso es de veinte años, que es el de vigencia de las patentes. 2) Podemos observar tres momentos de la evolución del sistema tecnológico: el inicial, el intermedio y el más reciente. 3) Los periodos se traslapan, lo cual permite mantener parte de la información de ellos y, de este modo, cierta continuidad en la evolución del sistema. 
la $701 / 22$, la 180/65.21 y la 318/139 (Electricity: motive power systems, batteryfed motor systems).

Gráfica 8. Red de clases de vehículos eléctricos para 1976-1995. Primera fase (conexión relativa)

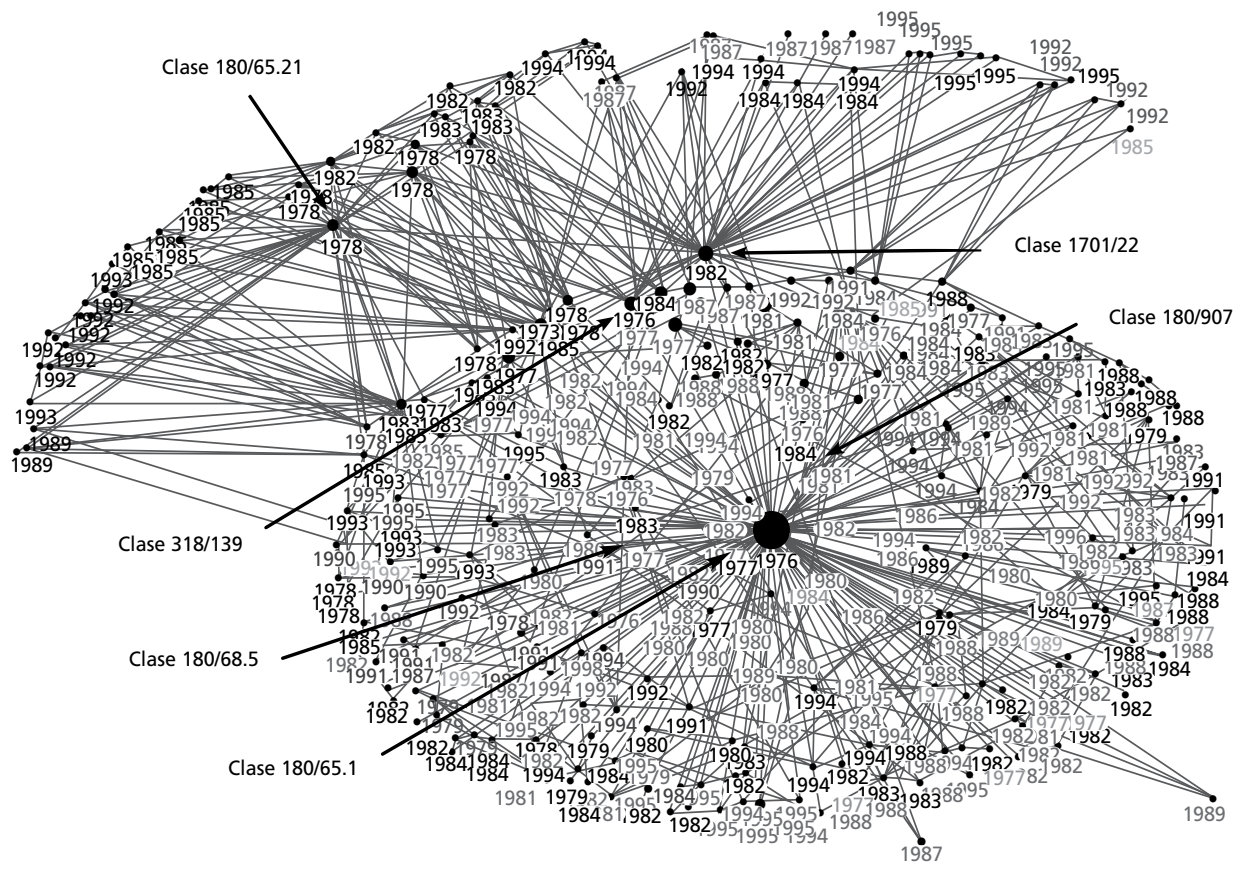

Fuente: Elaboración propia con información obtenida de la USPTO. Base de datos UAM/PECCI. Proyecto "Sistemas Complejos Adaptables y Cooperación Tecnológica", CONACYT No. 10017156204.

La integración de estas clases en un grupo que progresivamente se separa del resto del sistema y que mantiene al interior cierta autonomía se debe al hecho de que este conjunto de clases representa a los componentes claves de los vehículos eléctricos: la batería eléctrica, la tecnología híbrida que combina el motor de combustión interna con el eléctrico y el procesamiento de datos que permite administrar la energía del vehículo. En contraste con este avance, resaltan dos clases por su grado de conectividad: la clase 180/907 (Motor vehicles; motorized wheelchairs) y la clase 180/68.5 (Motor vehicles; power: battery mountings and holders). Son montables pequeños que muestran lo incipiente del desarrollo de los vehículos eléctricos durante este periodo e indican cierta desconexión en el sistema como conjunto. En resumen, en esta fase comienza a obser- 
varse cierto desacoplamiento de subsistemas que contrasta con una desconexión a nivel de sistema.

Gráfica 9. Red de clases de vehículos eléctricos para 1976-2001. Segunda fase (conexión y ordenamiento)

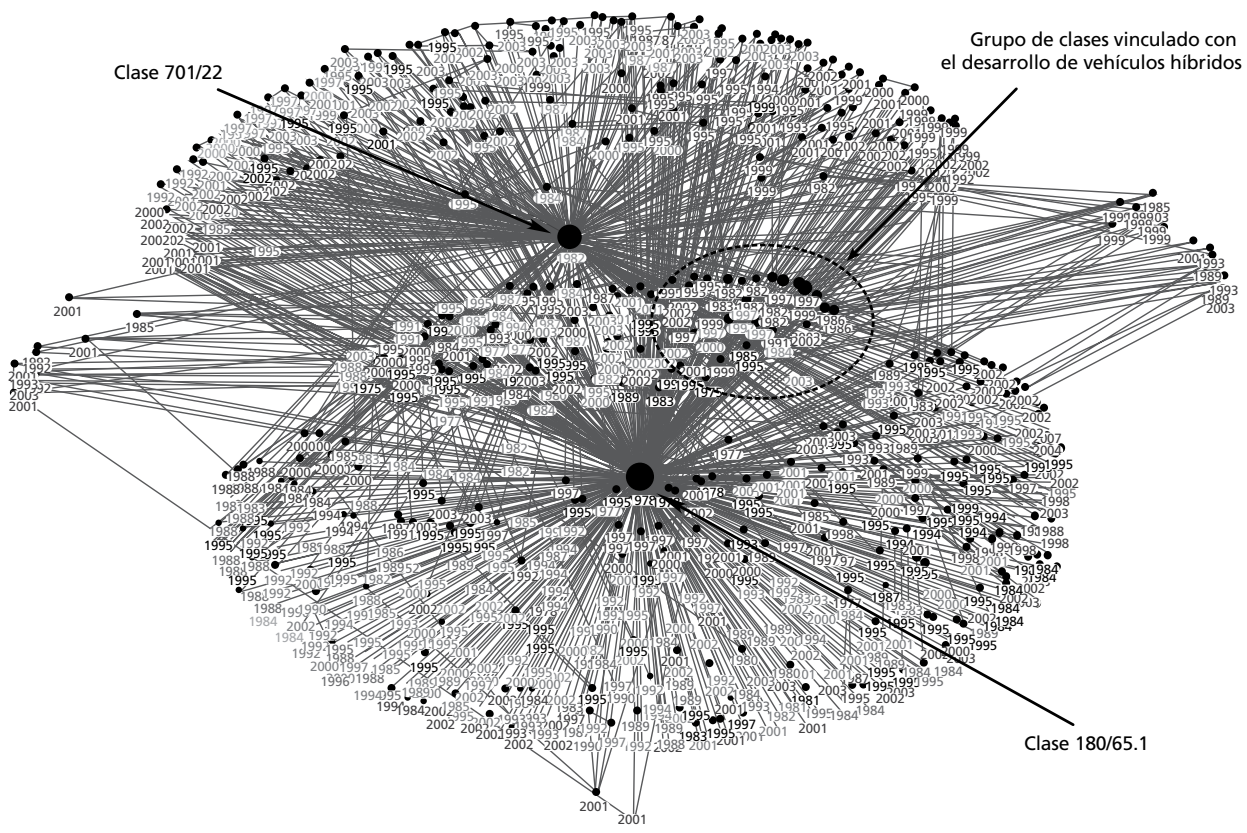

Fuente: Elaboración propia con información obtenida de la USPTO. Base de datos UAM/PECCI. Proyecto "Sistemas Complejos Adaptables y Cooperación Tecnológica", CONACYT No. 10017156204.

Segunda fase (1984-2003). La segunda fase de la evolución del sistema comprende tres distintos momentos: uno de baja actividad inventiva (1984-1990), otro que corresponde al periodo de despegue (1991-1994) y uno más en el que la actividad inventiva mostraba un crecimiento constante (1995-2000) (véase la gráfica 1). La red para esta fase muestra una clara separación de tres subsistemas, uno vinculado con la clase 180/65.1, el cual domina la conectividad del conjunto del sistema; otro, encabezado por la clase 701/22, y un conjunto de clases con grado de conectividad más homogéneo y que se vinculan con el desarrollo de vehículos híbridos ${ }^{31}$ (gráfica 9).

${ }^{31}$ Las primeras veinte clases de este grupo ordenadas según su grado de conectividad son: 903/903, 180/65.27, 180/65.285, 180/65.28, 180/65.29, 903/906, 903/947, 903/916, 903/951, 
Los grupos se encuentran conectados. Hay clases que se vinculan más con alguno de ellos, pero también hay clases que se relacionan con dos de los tres principales grupos e incluso con los tres. En esta fase, el grupo vinculado con la clase 180/65.1 tiene 1360 enlaces con el grupo de clases asociadas a la 701/22 y 1610 enlaces con el grupo de clases asociado al desarrollo de los vehículos eléctricos. A su vez, estos dos últimos grupos tienen 1088 enlaces. En resumen, los micropatrones generaron en el agregado del sistema una estructura de grupos o subsistemas claramente identificables y conectados entre sí.

Tercera fase (1993-2012). En la fase más reciente se incluyen los periodos de despegue y crecimiento acelerado en la actividad inventiva (véase la gráfica 1). Se observa una estructura similar a la de fase anterior (gráfica 10), sin embargo, se detectan dos elementos relevantes. El primero, el grupo de patentes que se asocian con el desarrollo de vehículos híbridos desplaza en importancia al grupo de clases vinculadas a la 701/22. Los grupos se intercambiaron en la segunda y la tercera posición. El segundo elemento es que el crecimiento de las clases contenidas en el grupo de las patentes asociadas con el desarrollo de vehículos híbridos se distribuye más claramente tanto en su grupo como en los otros dos grupos principales. Los enlaces del grupo de clases de los vehículos híbridos con el grupo asociado con la clase 180/65.1 ascienden a 5160 y a 5112 con el grupo de clases asociado a la 701/22, en tanto que entre estos dos últimos grupos sólo hay 1688 enlaces. Esto significa que los micropatrones de esta fase muestran que la red se ha enlazado de tal modo que se forman tres grandes grupos de clases conectados jerárquicamente entre ellos.

Una vez identificado este conjunto de micropatrones es posible describir la evolución del sistema de clases tecnológicas en su conjunto como resultado de las distintas formas de desacoplamiento-integración que se producen tanto en el nivel local, como en el total del sistema. ${ }^{32} \mathrm{Si}$ se asume que la información de las clases tecnológicas permite reconstruir los procesos de invención, esta perspectiva analítica es relevante en varios sentidos. En primer lugar, permite representar

903/910, 180/65.235, 180/65.25, 903/919, 180/65.245, 903/918, 903/946, 903/945, 180/65.6, $903 / 909$ y 180/65.26. Todas las que tienen 903 como sus primeros tres dígitos pertenecen a la clase Hybrid electric vehicles y el resto están anidadas en la 180/65.21 (Motor vehicles; electric power, hybrid vehicle).

${ }^{32}$ Este fenómeno es similar a la meiosis celular, ya que el sistema de invención, al igual que una célula, se reproduce con variaciones a partir de la recombinación genética interna (combinación de clases), el crecimiento y, finalmente, la separación. 
Gráfica 10. Red de clases de vehículos eléctricos para 2002-2012. Tercera fase (ordenamiento jerárquico)

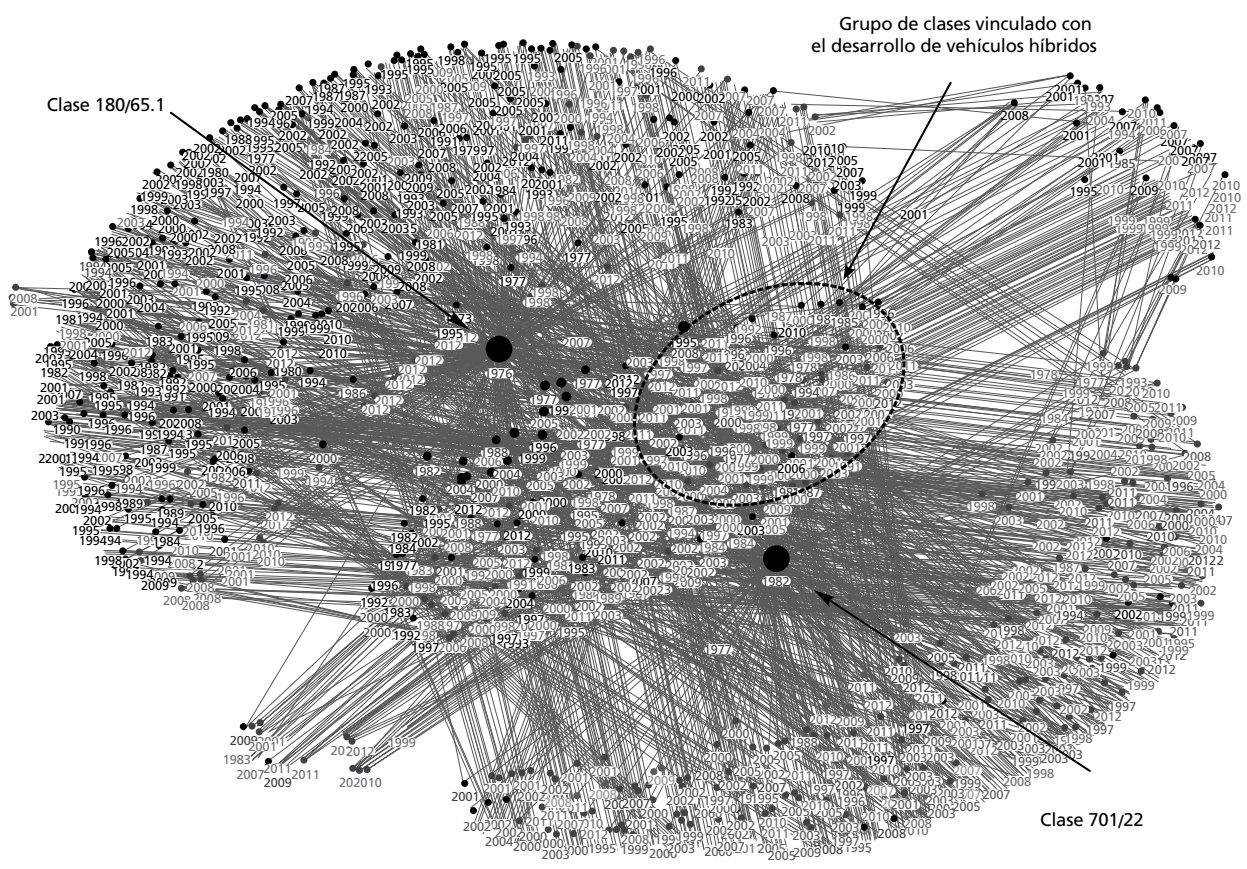

Fuente: Elaboración propia con información obtenida de la USPTO. Base de datos UAM/PECCI. Proyecto "Sistemas Complejos Adaptables y Cooperación Tecnológica", CONACYT No. 10017156204.

la complejidad de la actividad inventiva no como resultado del conocimiento individual de un agente o empresa, sino del conocimiento diverso asociado a un gran número de patentes y de agentes (véase la gráfica 2). En segundo lugar, a través de los procesos de agrupamiento y reagrupamiento de las clases tecnológicas es posible examinar las distintas trayectorias de evolución del conocimiento tecnológico. Y, por último, independientemente de la escala (subsistema o sistema), los micropatrones dan cuenta de los fenómenos de integración-desacoplamiento de las clases tecnológicas.

\section{b) Análisis cuantitativo de la evolución del sistema tecnológico}

Formación y dinámica de grupos. En el proceso de expansión de las clases tecnológicas, la integración de grupos de clases es un dato fundamental por dos razones. Primero, porque el número de grupos que se forman refleja el grado de 
conectividad de los subsistemas. Y, segundo, porque la posición en la que se encuentra cada grupo indica el grado de interés de los inventores por desarrollar determinado subsistema. En la gráfica 11 se puede observar cómo fluctúa el número de grupos. En la segunda mitad de los setenta se constituyeron dos y en 2005 se alcanzó un máximo de 73. Esto quiere decir que durante todo este periodo se aceleró la actividad de exploración de nuevas clases, lo que trae como consecuencia la diversificación de los agrupamientos y la creación de formas novedosas de conectividad entre los subgrupos. Sin embargo, en los años siguientes, la existencia de un número menor de grupos parece indicar la consolidación de determinados subsistemas.

Cabe preguntar, entonces, ¿cómo se comportan las clases al interior de los grupos? Para responder esta interrogante se tiene que observar: 1) la evolución en el número de clases que tiene cada grupo y 2) la permanencia o estabilidad de las clases en sus grupos.

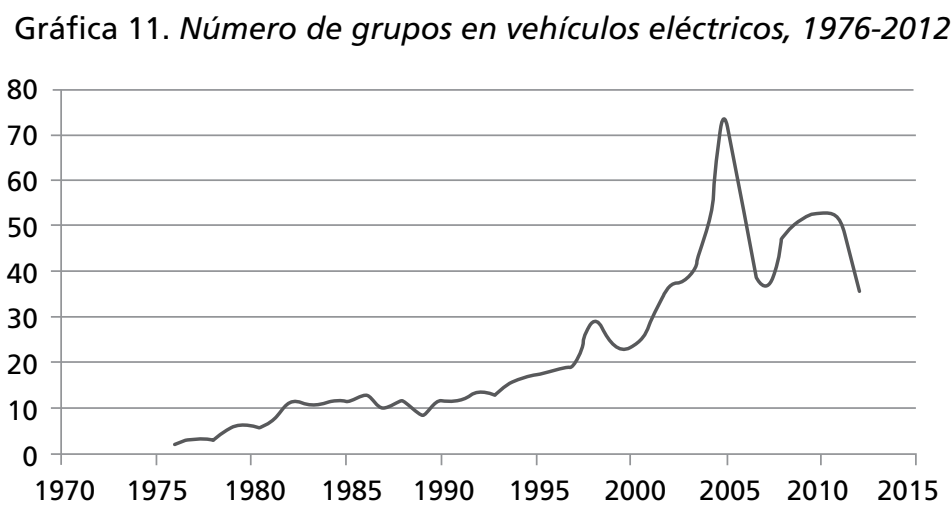

Fuente: Elaboración propia con información obtenida de la USPTO. Base de datos UAM/ PECCI. Proyecto "Sistemas Complejos Adaptables y Cooperación Tecnológica", CONACYT No. 10017-156204.

En la gráfica 12 se muestra cómo varía el número de clases al interior de los grupos. ${ }^{33}$ De esta gráfica se pueden destacar dos fenómenos. El primero es que en el periodo 1976-1995 la cantidad de clases para cada grupo tendió a converger: el número de clases de los cuatro grupos principales es más o menos similar en un rango que va de $12 \%$ a $20 \%$ de clases por grupo.

\footnotetext{
${ }^{33}$ Esto es, se contabiliza el número de clases (como porcentaje del total de clases) que contienen cada uno de los cuatro grupos más grandes.
} 
El segundo estriba en que en el periodo 1996-2012 se fueron consolidando tres grupos que, en conjunto, contenían $83 \%$ de las clases (32\% del total de clases para el primer grupo, $27 \%$ para el segundo y $23 \%$ para el tercero). La conformación de grupos con mayor peso en este último periodo, puede asociarse a la creación de bloques de construcción propios del sistema modular, en tanto que el primer periodo es posible vincularlo a la explosión de nuevas clases, propia del sistema integral.

\section{Gráfica 12. Evolución de los cuatro grupos principales de vehículos eléctricos,} 1976-2012

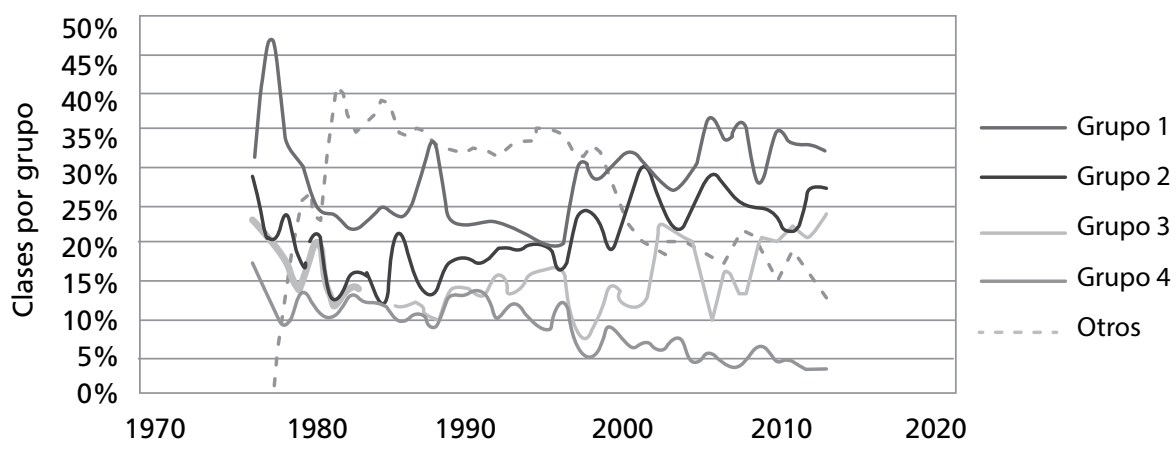

Fuente: Elaboración propia con información obtenida de la USPTO. Base de datos UAM/PECCI. Proyecto "Sistemas Complejos Adaptables y Cooperación Tecnológica", CONACYT No. 10017156204.

Respecto a la permanencia de las clases en cada subsistema, se observa un gran dinamismo. Prácticamente todas las clases han cambiado de grupo en algún momento. En el histograma de la gráfica 13 se indica la frecuencia con que las clases han cambiado de grupo, según rangos. Conforme el rango se acerca a 1 significa que las clases casi no han cambiado de grupo y si se acerca a 0 que se tendió a cambiar de grupo cada año. ${ }^{34}$ Se observa que sólo $0.002 \%$ de las clases

\footnotetext{
${ }^{34}$ El cálculo de este histograma se realizó de la siguiente forma: Si una clase cambia de grupo de un año al otro, se le asigna un 0 ; si no cambia, se le asigna un 1 . Se promedian los datos y se incluye en el rango decimal que le corresponde. Por ejemplo, la clase 60/275 perteneció sucesivamente a los grupos 22, 70, 47, 2, 29, 2, 3, 2 y 9 desde 2004 hasta 2012. Como todos los años cambió de grupo, por cada año se le asigna un 0 , el valor promedio es de 0 y se incluye en el rango que es mayor o igual 0 y menor a 0.1 . Por su parte, la clase $310 / 156.58$, en el mismo lapso perteneció a los grupos 19, 20, 25, 19, 26, 21, 21, 21 y 4, por lo que los valores asignados fueron $0,0,0,0,0,0,1,1$ y 0 , para un promedio de 0.2222 que la incluye en el rango de mayor o igual a 0.2 y menor a 0.3 .
} 
nunca han cambiado de grupo. ${ }^{35}$ Así, a pesar de que la dinámica de la red da cuenta de la consolidación de tres grandes áreas de especialización (33.66\% de las clases han cambiado relativamente poco de grupo), ${ }^{36}$ es notoria la poca estabilidad de las clases al interior de los grupos. Es decir, aunque a nivel de sistema se observa una tendencia relativamente estable, al interior de los grupos las clases transitan de un grupo a otro, movilidad propia de la introducción de nuevas clases y de sus múltiples relaciones.

\section{Gráfica 13. Histograma de variación de las clases entre grupos de vehículos eléctricos}

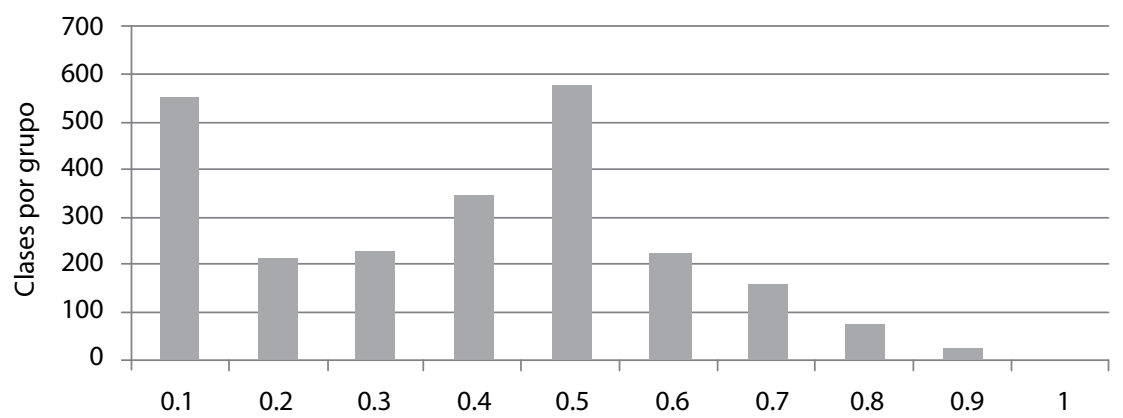

Fuente: Elaboración propia con información obtenida de la USPTO. Base de datos UAM/PECCI. Proyecto "Sistemas Complejos Adaptables y Cooperación Tecnológica", CONACYT No. 10017156204.

Con base en esta dinámica dual de las clases -estabilidad de los agrupamientos en grandes áreas (subsistemas) y alta movilidad al interior-, cabe preguntarse en qué medida el sistema en su conjunto tiende al orden o al desorden. Para determinar esto, se calcula la entropía ${ }^{37}$ anual que, en este caso, mide el grado de desorden del sistema según la interrelación de las clases tecnológicas. Desde 1976 hasta los primeros años de los ochenta se observa un incremento acelerado del desorden del sistema (gráfica 14). Este comportamiento se explica por la baja actividad inventiva del periodo, de tal manera que la inclusión de una nueva clase altera todo el sistema.

\footnotetext{
${ }^{35}$ De hecho, sólo las clases relativamente nuevas (con menos de cuatro años de existencia) presentan esta característica.

${ }^{36}$ Es importante notar que este cambio de grupo no necesariamente significa que una clase haya cambiado de un grupo a otro, sino que también es posible que todo el grupo haya cambiado de posición relativa respecto al conjunto de sistema.

${ }^{37}$ La entropía mide el grado de desorden de un sistema. Si la entropía es alta, significa que el sistema está desordenado; si la entropía es baja, significa lo contrario. Para este análisis se utilizó la noción de entropía de Shannon (Page, 2011).
} 
Gráfica 14. Entropía en el sistema de clases de los vehículos eléctricos

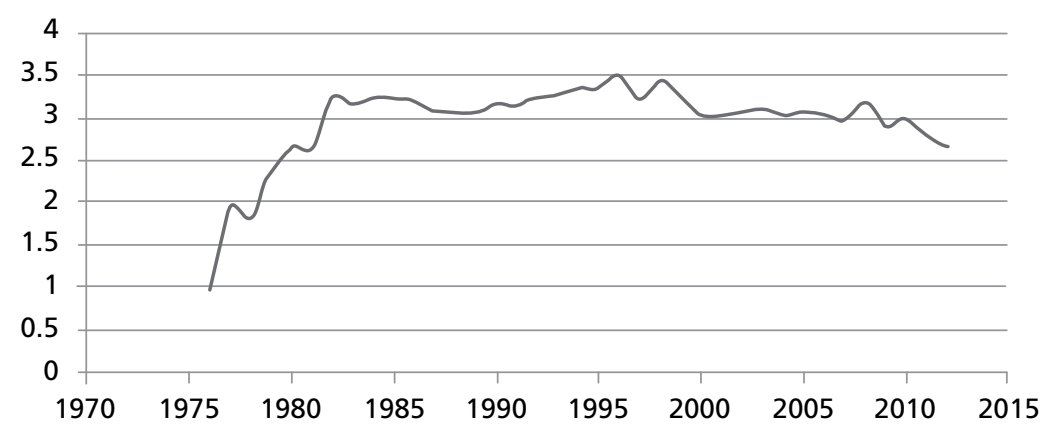

Fuente: Elaboración propia con información obtenida de la USPTO. Base de datos UAM/PECCI. Proyecto "Sistemas Complejos Adaptables y Cooperación Tecnológica", CONACYT No. 10017156204.

En los años siguientes de la década de los ochenta y la primera mitad de los noventa, el nivel de entropía fue alto pero relativamente estable, hasta llegar a un máximo global de 3.48 en $1996 .{ }^{38}$ Sin embargo, a partir del año 2000 hasta la fecha, la entropía disminuyó sistemáticamente a pesar del gran número de patentes que se registraron en este periodo. La razón de este fenómeno es que el sistema se ha ordenado en los diferentes subsistemas, como se describió más arriba. De esta forma, se ha pasado de una red pequeña y desconectada temporalmente a una red más conectada y ordenada (gráfica 14).

Estructura del sistema. El comportamiento que se analizó gráficamente arriba tiene su correlato cuantitativo sintetizado en la tabla 1. Esta información permite examinar la evolución y estructura de la red de clases. El número de nodos corresponde al número de clases $(N)$, cuyo crecimiento en el periodo 1976-2012 es exponencial; lo mismo ocurre con los enlaces $(k)$. En principio, los datos muestran que los diámetros ${ }^{39}$ de la red son significativos. En los primeros años (1976 a 1977), la red está muy conectada (su diámetro es de 2) debido a las pocas patentes de ese periodo. De 1978 a 1992 la red tiende a desconectarse, su diámetro pasa de 3 a 4, aunque a partir de 1993 y hasta el año $2012^{40}$ la red se vuelve a

\footnotetext{
${ }^{38}$ De 1992 en adelante se inicia una intensa actividad inventiva; es un periodo en el que la red pasó de estar relativamente desconectada a convertirse en una red altamente conectada.

${ }^{39}$ El diámetro de una red es el camino más largo de los caminos más cortos que conectan cualquiera de los nodos o, en otras palabras, el número mínimo de nodos por los que se tiene que pasar, incluyendo los extremos, para conectar a los nodos más lejanos. Brinda una idea de la conectividad de una red.

${ }^{40}$ Que coincide con la explosión en la actividad inventiva.
} 
Tabla 1. Estadísticos de las redes de los vehículos eléctricos, 1976-2012

\begin{tabular}{|c|c|c|c|c|c|c|c|c|c|c|c|}
\hline Año & Nodos & $\begin{array}{l}\text { Enlaces } \\
\text { únicos }\end{array}$ & $\begin{array}{c}\text { Enlaces } \\
\text { duplicados }\end{array}$ & \begin{tabular}{|c|}
$\begin{array}{c}\text { Total } \\
\text { de } \\
\text { enlaces }\end{array}$ \\
\end{tabular} & $\begin{array}{l}\text { Nodos/ } \\
\text { Enlaces }\end{array}$ & ito-loops & siámetro & \begin{tabular}{|l|} 
Distancia \\
geodésica \\
promedio
\end{tabular} & Grupos & Densidad & Modularidad \\
\hline 1976 & 8 & 16 & 0 & 16 & 0.5 & 0 & 2 & 1.25 & 2 & 0.571428571 & 0.25 \\
\hline 1977 & 30 & 85 & 9 & 94 & 0.352941 & 0 & 2 & 1.735556 & 4 & 0.204597701 & 0.453259 \\
\hline 1978 & 49 & 170 & 48 & 218 & 0.288235 & 0 & 3 & 1.96918 & 4 & 0.159863946 & 0.430267 \\
\hline 1979 & 63 & 208 & 41 & 249 & 0.302885 & 2 & 3 & 2.025699 & 6 & 0.115719406 & 0.48352 \\
\hline 1980 & 73 & 251 & 52 & 303 & 0.290837 & 2 & 3 & 2.034903 & 7 & 0.104261796 & 0.494834 \\
\hline 1981 & 94 & 333 & 58 & 391 & 0.282282 & 2 & 3 & 2.044817 & 7 & 0.081903455 & 0.542813 \\
\hline 1982 & 143 & 527 & 80 & 607 & 0.271347 & 2 & 3 & 2.092327 & 11 & 0.055254605 & 0.588207 \\
\hline 1983 & 160 & 577 & 84 & 661 & 0.277296 & 2 & 3 & 2.085469 & 11 & 0.048113208 & 0.582982 \\
\hline 1984 & 188 & 708 & 99 & 807 & 0.265537 & 2 & 3 & 2.096084 & 12 & 0.042610081 & 0.579435 \\
\hline 1985 & 198 & 729 & 103 & 832 & 0.271605 & 2 & 4 & 2.170442 & 12 & 0.039583654 & 0.587072 \\
\hline 1986 & 209 & 777 & 117 & 894 & 0.268983 & 2 & 4 & 217165 & 13 & 472 & 873 \\
\hline 1987 & 223 & 858 & 131 & 989 & 0.259907 & 2 & 4 & 7036 & 11 & 418 & 135 \\
\hline 1988 & 253 & 996 & 153 & 1149 & 0.254016 & 2 & 4 & 2.261994 & 12 & 9033 & 349 \\
\hline 1989 & 271 & 1056 & 185 & 1241 & 0.256629 & 2 & 4 & 2.27151 & 10 & 0.030859642 & 0.583661 \\
\hline 1990 & 280 & 1086 & 281 & 1367 & 0.257827 & 2 & 4 & 57423 & 12 & 0.030363543 & 0.547263 \\
\hline 1991 & 297 & 1132 & 328 & 1460 & 0.262367 & 2 & 4 & 216327 & 12 & 01028 & 0.537372 \\
\hline 1992 & 336 & 1327 & 400 & 1727 & 3203 & 2 & 4 & 493 & 14 & 2878 & 947 \\
\hline 1993 & 356 & 1431 & 498 & 1929 & 0.248777 & 2 & 3 & 2.209964 & 14 & 02522551 & 0.487046 \\
\hline 1994 & 402 & 1656 & 635 & 2291 & 0.242754 & 2 & 3 & 2.197842 & 17 & 0.023051823 & 0.479149 \\
\hline 1995 & 441 & 1776 & 804 & 2580 & 0.248311 & 2 & 3 & 08997 & 18 & 0.020902907 & 507 \\
\hline 1996 & 535 & 2232 & 1023 & 3255 & 0.239695 & 2 & 3 & 2.193261 & 19 & 0.017858518 & 203 \\
\hline 1997 & 585 & 2479 & 1105 & 3584 & 0.235982 & 3 & 3 & 2.221866 & 20 & 96897 & 099 \\
\hline 1998 & 662 & 2908 & 1442 & 4350 & 0.227648 & 3 & 3 & 14 & 30 & 015302275 & 0.44 \\
\hline 1999 & 744 & 3617 & 2758 & 6375 & 0.205695 & 3 & 3 & 2.263647 & 24 & 0.015680401 & 0.416215 \\
\hline 2000 & 811 & 4068 & 3296 & 7364 & 0.199361 & 3 & 3 & 2.270525 & 24 & 0.014948775 & 0.399082 \\
\hline 2001 & 910 & 4943 & 4426 & 9369 & 0.184099 & 4 & 3 & 2.272018 & 29 & 0.014584316 & 0.348095 \\
\hline 2002 & 1082 & 6709 & 6456 & 13165 & 0.161276 & 5 & 3 & 2.264341 & 37 & 0.013995735 & 0.344776 \\
\hline 2003 & 1230 & 7789 & 8121 & 15910 & 0.157915 & 6 & 3 & 2.291008 & 39 & 0.012570204 & 0.339868 \\
\hline 2004 & 1294 & 8325 & 9338 & 17663 & 0.155435 & 7 & 3 & 2.286256 & 50 & 0.012208169 & 0.318373 \\
\hline 2005 & 1392 & 9021 & 10409 & 19430 & 0.154307 & 9 & 3 & 2.279286 & 73 & 0.011487022 & 0.327287 \\
\hline 2006 & 1473 & 9623 & 11210 & 20833 & 0.153071 & 10 & 3 & 2.272887 & 50 & 0.010941512 & 0.326016 \\
\hline 2007 & 1587 & 10396 & 11979 & 22375 & 0.152655 & 11 & 3 & 2.287462 & 37 & 0.010147073 & 0.335135 \\
\hline 2008 & 1662 & 11069 & 12982 & 24051 & 0.150149 & 15 & 3 & 2.278327 & 47 & 0.009893566 & 0.318214 \\
\hline 2009 & 1800 & 12348 & 14658 & 27006 & 0.145773 & 17 & 3 & 2.270465 & 51 & 0.00948984 & 0.329608 \\
\hline 2010 & 1922 & 13682 & 16634 & 30316 & 0.140477 & 24 & 3 & 2.280906 & 53 & 0.009272074 & 0.316975 \\
\hline 2011 & 2124 & 15957 & 19115 & 35072 & 0.133108 & 33 & 3 & 2.281611 & 51 & 0.008828072 & 0.311535 \\
\hline 2012 & 2394 & 19669 & 23895 & 43564 & 0.121714 & 57 & 3 & 2.277313 & 36 & 0.008603135 & 0.303294 \\
\hline
\end{tabular}

Nota: Cabe señalar que la distancia geodésica promedio se refiere a la media de los diámetros e indica qué tan cerca se encuentran los nodos en promedio. La densidad es la porción del total de enlaces existentes respecto al total de los potenciales e indica qué tan conectados están respecto a un escenario donde todos los nodos estén conectados entre sí directamente. La modularidad es la porción de enlaces que entran en un grupo dado, menos la fracción esperada si los enlaces estuvieran distribuidos aleatoriamente, lo que permite determinar si la estructura de la red es uniforme (un solo gran componente) o está constituida por grupos.

Fuente: Elaboración propia con información obtenida de la USPTO. Base de datos UAM/PECCI. Proyecto "Sistemas Complejos Adaptables y Cooperación Tecnológica", CONACYT No. 10017-156204. 
conectar (un diámetro de 3 con distancias geodésicas promedio mayores). Esta mayor conectividad se produce a pesar del explosivo crecimiento de la red y si se contrastan estos resultados con el nivel de densidad y de "modularidad"4l se observa que, a pesar de que el volumen combinatorio de las clases es muy alto (2.86 millones de posibles combinaciones por pares), sólo se exploran 43000 combinaciones. Es decir, no todo el espacio de soluciones posibles es explorado, sino sólo un conjunto bien delimitado, que se halla asociado a la naturaleza de los problemas tecnológicos que enfrentan los agentes. Por esta razón, se puede señalar que el espacio de exploración de clases tecnológicas en los vehículos eléctricos se encuentra ordenado.

Este mismo fenómeno se observa con los grupos, sólo que en ellos, el diámetro y la densidad son mayores. Es decir, hay mayor desconexión entre los grupos, aunque dentro de cada uno se explora más, lo cual es un indicador de desacoplamiento interno. ${ }^{42}$ De esta manera, se hacen notorios dos aspectos del fenómeno: primero, que al interior de los subsistemas se incrementa la complejidad y, en segundo lugar, que se conforma una estructura ordenada de subsistemas especializados.

Por tanto, es posible identificar cuatro subsistemas permanentes o estables a partir de 1995: 1) Diseño y desarrollo del motor eléctrico (clase 180/65.1). 2) Procesamiento de datos y administración de energía (clase 701/22). 3) Vehículos híbridos (principalmente las clases 180/65.21 y 903/903). 4) Otros vehículos eléctricos (sillas de ruedas y montables pequeños alrededor de la clase 180/907). Estos subsistemas se encuentran interconectados de tal modo que configuran una red de invención ordenada y jerárquica.

Hasta aquí, se puede señalar que las patentes encapsulan áreas de conocimiento (clases) que brindan solución a problemas tecnológicos concretos. Con la acumulación de estas soluciones se forman micropatrones en los que se vinculan las distintas áreas de conocimiento con diversos grados de conectividad. Durante este proceso emergen actividades inventivas que tienden a converger (inicialmente en torno al desarrollo del motor eléctrico) y a dar lugar a la aparición de agrupaciones o subsistemas. Así, con el transcurso del tiempo se crean soluciones que forman nuevos espacios cada vez más relevantes, de tal forma que la red de conocimiento evoluciona de manera dinámica y compleja, pero or-

\footnotetext{
${ }^{41}$ Los cuales indican los espacios posibles donde la red puede conectarse (véase tabla 1).

${ }^{42} \mathrm{Sin}$ embargo existen diferencias significativas de grupo a grupo. El grupo principal está más conectado internamente que el grupo subsiguiente en importancia.
} 
denada, en espacios particulares (motor eléctrico, procesamiento de datos de los diversos subsistemas del vehículo y desarrollo de vehículos híbridos) e interconectados, formando una jerarquía de subsistemas. En la siguiente sección se explora la relación entre la complejidad de las clases tecnológicas y la existencia de patentes traslapadas y de los anticomunes.

\section{COMPLEJIDAD, PATENTES TRASLAPADAS Y ANTICOMUNES EN LOS VEHÍCULOS ELÉCTRICOS}

Los resultados anteriores ilustran la naturaleza y la evolución compleja de los vehículos eléctricos. Sin embargo, cabe preguntar si existe relación entre esta complejidad y la posible existencia de patentes traslapadas. En particular, si existe relación entre la conectividad de las clases (áreas de conocimiento) y el número de agentes que participan en cada clase. Por ello, el grado de conectividad de cada clase adquiere una nueva importancia. Si el grado de conectividad de una clase (el número de clases con las que se conecta) es bajo o si el grado de conectividad es muy alto pero hay pocos agentes, entonces es probable que no haya patentes traslapadas. Por el contrario, si el grado de conectividad es alto y hay muchos agentes, es mayor la probabilidad de que existan.

Veamos la evidencia. En la actividad inventiva de los vehículos eléctricos, participan 411 agentes distintos. ${ }^{43}$ Esto quiere decir que cada uno se relaciona con 5.82 clases en promedio. Sin embargo, la distribución no es normal, es una distribución de la ley de potencia: muy pocas clases concentran muchos agentes ( $o$, alternativamente, muchos agentes se concentran en pocas clases) y muchas clases poseen pocos agentes. Por ejemplo, la clase 180/65.1 tiene 311 agentes $(75.66 \%$ del total) mientras que 1499 clases $(62.61 \%$ de ellas) sólo cuentan con uno. La media de esta distribución es de 2.98 , que resulta, en términos de patentes traslapadas, un número relativamente bajo. Por lo tanto, si consideramos sólo este indicador, cada agente tendría que negociar con un máximo de tres agentes adicionales para cubrir el total de patentes de una clase.

Sin embargo, la evidencia señala que existe una fuerte relación $\left(R^{2}=0.967\right)$ entre el grado de conectividad de una clase y el número de agentes que participan en ella (gráfica 15). La razón de este fenómeno estriba en que los

\footnotetext{
${ }^{43}$ Que pueden ser empresas, centros de investigación (públicos o privados), universidades (públicas o privadas), organizaciones gubernamentales o inventores independientes (considerados en nuestro análisis como un solo agente).
} 
desarrolladores de vehículos eléctricos enfrentan numerosos problemas, algunos de ellos relativamente simples y otros muy complejos. ${ }^{44}$ Cuando el problema es complejo: 1) ningún agente puede resolverlo totalmente (por lo menos en el corto plazo); 2) el problema se divide en subproblemas más simples, los cuales sí pueden ser tratados, y 3) las soluciones a cada subproblema son complementarias. Así, en el nivel del subproblema se presentan dos casos: a) el surgimiento de múltiples soluciones a partir de la exploración de diversos campos tecnológicos o b) el surgimiento de múltiples soluciones a partir de un conjunto común de clases.

Estas dos posibilidades implican que un subproblema puede ser resuelto por muchos grupos de clases distintos y que un mismo grupo de clases puede resolver distintos subproblemas. De este modo, las clases se conectan de acuerdo al grado de complementariedad de sus subproblemas. Los agentes seleccionan los problemas y las áreas tecnológicas (clases) que pueden resolver de acuerdo con sus capacidades e intereses. En particular, las empresas automotrices ensambladoras patentan una parte pequeña del total de clases: sólo $11.48 \%$, pero en clases con grados de conectividad promedio de 66.6. Esto es, tres veces más que el promedio general, que es de $20.5 .{ }^{45}$ En todos estos procesos, la posibilidad de que la naturaleza compleja del problema conduzca a la conformación de una densa red de clases y derechos de propiedad traslapados es mayor.

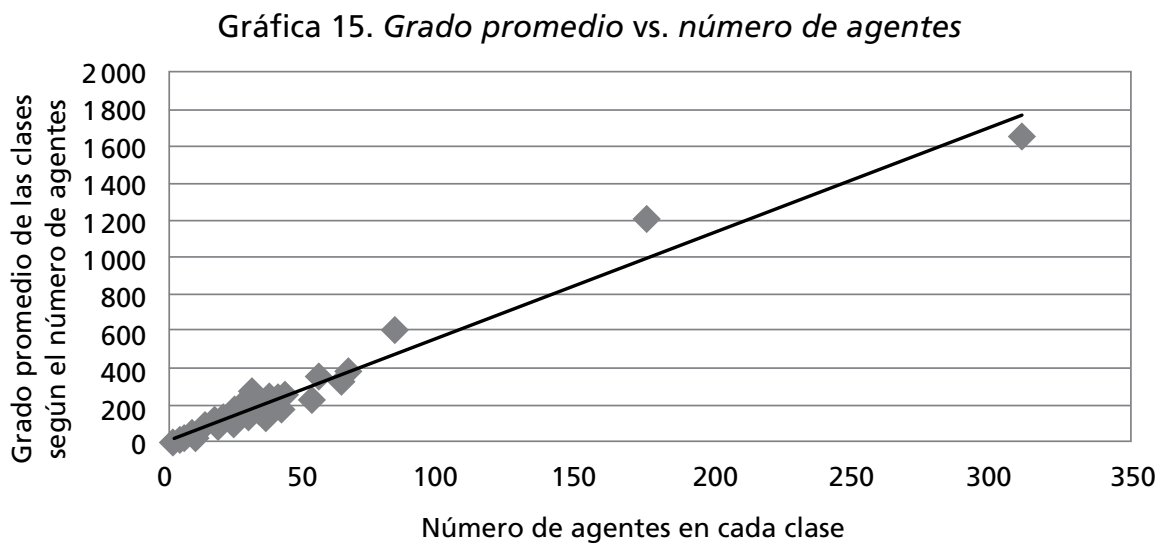

Fuente: Elaboración propia con información obtenida de la USPTO. Base de datos UAM/PECCI. Proyecto "Sistemas Complejos Adaptables y Cooperación Tecnológica", CONACYT No. 10017156204

\footnotetext{
${ }^{44}$ Por ejemplo, el desarrollo del motor eléctrico o el sistema de acumulación de energía (baterías).

${ }^{45}$ Fuente de la información: Base de datos UAM/PECCI. Proyecto "Sistemas Complejos Adaptables y Cooperación Tecnológica”, CONACYT No. I0017-156204.
} 


\section{CONCLUSIONeS}

Para indagar sobre la existencia de patentes traslapadas en el diseño y desarrollo de los vehículos eléctricos, durante el periodo 1974-2012, este trabajo propone, integrando la teoría de Ostrom y la teoría de los sistemas complejos, una forma de representar la evolución del conocimiento tecnológico. A partir de la información que brindan las patentes -en particular la relacionada con las clases tecnológicas-, se reconstruye la evolución tecnológica mediante la cual el conocimiento tecnológico se integra-desacopla en subsistemas cada vez más complejos. A continuación, se resumen los principales hallazgos.

La evidencia permite señalar que en la evolución del conocimiento tecnológico de los vehículos eléctricos se presentan por lo menos seis momentos a lo largo del periodo analizado. En el lapso de 1976 a 1982, la actividad inventiva es baja (41 patentes), con una red de conocimiento/clases desconectada, de tal forma que cualquier incorporación de una nueva clase a la red incrementa la entropía de ésta. De 1983 a 1990, la red de conocimiento prácticamente no se modifica (54 patentes registradas). Estos dos primeros momentos pueden caracterizarse como una fase de escasa actividad inventiva, con una red pequeña y relativamente desconectada.

A partir de 1991 y hasta 1995, la actividad inventiva se incrementa (92 patentes), pero sólo en algunas áreas de investigación -233 clases, en especial, las vinculadas al desarrollo del motor eléctrico y de vehículos pequeñosrelativamente desconectadas entre sí. De 1996 a 2000, la actividad inventiva y los espacios de exploración de soluciones se expanden (235 patentes y 510 clases). Fundamentalmente, se incrementa el peso de la clase relacionada con el procesamiento de datos. Durante estos dos periodos, la red de clases crece y se conecta más.

De 2001 a 2005, se presenta un fenómeno significativo: aumenta el número de patentes (504), de clases (879) y de subsistemas (de 29 en 2001 a 73 en 2005), pero el nivel de entropía no (con un mínimo de 3.02 y un máximo de 3.1). Es un periodo en el que comienzan a emerger los subsistemas más definidos, pero sin plena integración. La red empieza a ordenarse y de 2005 a 2012 se presentan tres fenómenos. El primero es que el número de subsistemas se reduce (de 73 a 36) y también el nivel de entropía (de 3.06 a 2.66). El segundo consiste en que el subsistema de procesamiento de datos mantiene su tendencia creciente y su autonomía relativa con respecto del sistema (con un grado de conectividad de 943 para la clase 701/22, segunda más conectada), pero pasa al cuarto lugar 
de importancia relativa de los grupos. Y el tercero se refiere a que el conjunto de patentes relacionadas con el desarrollo del vehículo híbrido tiene mayor número de conexiones entre los subsistemas principales (9407 conexiones con los cuatro grupos más relevantes). En conjunto, estos fenómenos implican que la red adquiere un mayor orden jerárquico. En resumen, la red de conocimiento de los vehículos eléctricos pasa de ser pequeña y relativamente desconectada (1978-1990), a estar más conectada (1991-2000) y, finalmente, se convierte en una red conectada jerárquicamente (2001-2012).

Una de las virtudes de la teoría de redes es que permite reconstruir la complejidad de la evolución del conocimiento tecnológico. Es útil para explicar cómo el proceso de incorporación de nuevas clases tecnológicas en la red de clases se produce siguiendo micropatrones. $\mathrm{Y}$ es a través de la existencia de diferentes micropatrones que se puede explicar la emergencia de los subgrupos o subsistemas tecnológicos.

En la medida en que cada uno de los subsistemas tiene, internamente, un mayor número de interacciones con el resto de los subsistemas, adquiere la posibilidad de diferenciarse, consolidarse e incluso dominar el conjunto del sistema. Esta descripción sintetiza la relación que en un sistema tecnológico complejo se establece entre los modelos de diseño integral y modular. En el estudio, se identifica y se describe la emergencia de tres subsistemas relevantes en los vehículos eléctricos: el asociado con el desarrollo del motor eléctrico (actualmente dominante), el asociado con el procesamiento de datos (el cual posiblemente dominará a la industria) y el asociado con el desarrollo de los vehículos híbridos (que en los últimos años ha adquirido mayor importancia).

Es justo en estos subsistemas donde se observa simultáneamente una alta conectividad entre las clases y una mayor densidad (población) de agentes participantes, lo cual es un claro indicador de la presencia de patentes traslapadas. De hecho, se demuestra que en el caso de la actividad inventiva de los vehículos eléctricos, hay una relación directa entre el grado de conectividad de las clases y el número de agentes que participan en ellas. En toda la red existen 411 agentes: empresas, alianzas, universidades, centros de investigación, gobiernos o inventores individuales. Esta diversidad implica que también se establecen diversos tipos de relaciones entre los agentes, sin embargo, los datos indican que en $11.48 \%$ de las clases que se caracterizan por tener un alto grado de conectividad (66.62) hay patentes de empresas automotrices rivales. Así, es posible afirmar que en la actividad inventiva en el campo de los vehículos eléctricos existe conocimiento complementario (alta conectividad) y conocimiento fragmentado 
(en propiedad de diversas empresas automotrices rivales), lo que indica un escenario de anticomunes. La solución requiere de arreglos institucionales novedosos que eviten el mutuo bloqueo entre las empresas.

Después de este recorrido, se puede señalar que la teoría de los sistemas complejos permite identificar la existencia y evolución de una densa red de conocimientos tecnológicos, propiedad intelectual y agentes en el desarrollo de los vehículos eléctricos. La técnica de la teoría de redes y, en particular, la existencia de medidas cuantitativas de la evolución de la red permiten describir con mayor rigor cuál es la relación de la naturaleza del bien con la presencia de patentes traslapadas y el problema de los anticomunes.

\section{ANEXo METOdológico}

Los pasos generales que se siguieron para la construcción de las redes de este trabajo fueron:

a) Del sistema de información de patentes de la USTPO (http://patft.uspto. gov/netahtml/PTO/search-adv.htm) se descargaron las patentes del 1 de enero de 1976 al 20 de noviembre de 2012 correspondientes a las clases tecnológicas $180 / 65.1,180 / 65.21$ y 701/22 por medio del comando de búsqueda (ccl/180/65.1 or ccl/180/65.21 or ccl/701/22).

b) Se extrajeron todas las clases tecnológicas y se ordenaron en una nueva base.

c) Con los datos de dicha base, se construyó la matriz de adyacencia de las clases vinculadas por patentes. Es decir, para cada par de clases se asignaba un vínculo si una patente contenía dicho par de clases.

d) Se introdujo esta matriz en el programa de análisis de redes NodexL, anexando una columna en la que se especificaba el año que por primera vez se patentó cada clase.

e) Se generaron las redes y sus estadísticos para su análisis posterior.

\section{REFERENCIAS BIBLIOGRÁFICAS}

Baldwin, Carliss Y., y Clark, Kim B. (1999), Design Rules, Massachusetts, мIT Press. Ballardini, Ros M. (2009), "The Software Patent Thicket: a Matter of Disclousure", sCRIPTed, 6 (2), pp. 208-233. 
Bessen, James; Ford, Jennifer, y Meurer, Michael J. (2012), "The Private and Social Costs of Patent Trolls", Regulation, 4 (34), pp. 26-35.

Bessen, James, y Maurer, Michael J. (2008), Patent Failure: How Judges, Bureaucrats and Lawyers Put Innovators at Risk, New Jersey, Princenton University Press.

Clauset, Aaron; Newman, Mark E. J., y Moore, Christopher (2004), "Finding Community Structure in Very Large Networks", Physical Review E, 70, 066111.

Clarkson, Gavin, y DeKorte, David (2006), "The problem of Patent Thickets in Convergent Technologies", Annals of New York Academic of Sciences, 1093 (0), pp. 180-200.

Coriat, Benjamin (2011), "From Natural-Resource Commons to Knowledge Commons. Common Traits and Differences", LEM Working Paper Series 16, pp. 1-26.

Cowan, Robin, y Hultén, Staffan (1996), "Escaping Lock-in: The Case of the Electric Vehicle”, Technological Forecasting and Social Change, 53 (1), pp. 61-79.

Fixon, Sebastian, y Sako, Mari (2001), "Modularity in product architecture: Will the Auto Industry Follow the Computer Industry? (An analisys of Product Architecture, Market Conditions, and Institutional Forces)", ponencia a la Fall Meeting 2001 International Motor Vehicle Program (IMVP), realizada en Cambridge, MA, el 10 y 11 de septiembre.

Frenken, Koen; Marengo, Luigi, y Valente, Marco (1999), "Interdependencies, NearlyDecomposobality and Adaptacion", en Thomas Brenner (ed.), Computational Techniques for Modelling Learning in Economics, NY,Springer, pp. 145-165.

Graevenitz, Georg von; Wagner, Stefan, y Harhoff, Dietmar (2011), "How to Measure Patent Thickets: a Novel Approach”, Economics Letters, 111 (1), pp. 6-9.

Heller, Michael A. (1998), "The Tragedy of the Anticommons: Property in the Transition from Marx to Markets”, Harvard Law Review, 111 (3), pp. 621-688. (2008), The Gridlock Economy, New York, Basic Books.

Heller, Michael A., y Eisenberg, Rebecca S. (1998), "Can Patents Deter Innovation? The Anticommons in Biomedical Research”, Science, 280 (5364), pp. 698-701.

Hess, Charlotte, y Ostrom, Elinor (2003), "Ideas, Artifacts, and Facilities: Information as a Common-Pool Resource", Law and Contemporary Problems, 16 (111), pp. 111-145.

Holland, John H. (1996), El orden oculto, de cómo la adaptación crea la complejidad, México, FCE.

IPO (2011), Patent thickets. Team Intellectual Property Office Patent Informatics, Newport, Intellectual Property Office.

Juliussen, Egil, y Robinson, Richard (2010), Is Europe in the Driver's Seat? The Compe- 
titiveness of the European Automotive Embedded Systems Industry, Londres, Institute for Prospective Technological Studies,/European Comission.

Kauffman, Stuart A. (1993), The Origins of Order: Self-Organization and Selection in Evolution, New York, Oxford University Press.

Langlois, Richard N. (2002), "Modularity in Technology and Organization", Journal of Economic Behavior \& Organization, 9 (1), pp. 19-37.

Lara, Arturo (2014), "From Complex Mechanical System to Complex Electronic System: The Case of Auto-mobiles", International Journal of Automotive Technology and Management, 14 (1), pp. 65-81.

Lara, Arturo, y García, Alejandro (2005), "Coordinación interfirma y cercanía geográfica. El caso de Volkswagen-Puebla”, en A. Lara, H. Juárez y C. Bueno (coords.), El auto global. Desarrollo, competencia y cooperación en la industria del automóvil, Puebla, BUAP/UAM-X/Ui/CONACYT, pp. 431-455.

Lara, Arturo, y Salazar, César E. (2013), "Complejidad, instituciones y trayectoria tecnológica del automóvil: El caso de la batería de plomo-ácido", en F. Novelo (coord.), El retorno del desarrollo, México, UAM-X, pp. 101-121.

Mandelbrot, Benoit (1997), La geometría fractal de la naturaleza, Barcelona, Tusquets Editores.

National Research Council (2013), Transitions to Alternative Vehicles and Fuels, Washington, DC, The National Academies Press.

Osorio, Helder, y Lara, Arturo (en prensa), "The tragedy of the Anticommons in the construction of knowledge of the Human Genome", International Journal of the Commons.

Ostrom, Elinor (1990), Governing the Commons: The Evolution of Institutions for Collective Acction, New York, Cambridge University Press. (2005), Understanding Institutional Diversity, Princenton, Pricenton Unversity Press.

Overwalle, Geertrui van (2009), Gene Patents and Collaborative Licensing Models: Patent Pools, Clearinghouses, Open Source Models and Liability Regimes, Cambridge, Cambridge University Press.

Page, Scott E. (2011), Diversity and Complexity, Princenton, Princenton University Press.

Pandremenos, John; Paralikas, John; Salonitis, Konstantinos, y Chryssolouris, George (2009), "Modularity Concepts for the Automotive Industry: A critical Review", CIRP Journal of Manufacturing Science and Technology, 1 (3), pp. 148-152.

Poteete, Amy R.; Janssen, Marco A., y Ostrom, Elinor (2011), Working Together: Co- 
llective Action, the Commons, and Multiple Methods in Practice, Princeton, Princeton University Press.

Reyes, Juan (2012), "Diversidad y complejidad en el sector de baterías en el periodo 1976-2010", México, tesis doctoral por la UAM.

Sanchez, Ron, y Mahoney, Joseph T. (2003), "Modularity, Flexibility, and Knowledge Management in Product and Organization Design", en Raghu Garud, Arun Kumaraswamy y Richard N. Langlois (eds.), Managing in the Modular Age. Architectures, Networks and Organizations, Oxford, Blackwell Publishers, pp. 362-389.

Shapiro, Carl (2001), "Navigating the Patent Thicket: Cross Licenses, Patent Pools, and Standard-Setting", en Adam B. Jaffe, Josh Lerner y Scott Stern, Innovation Policy and the Economy, Vol 1, Cambridge, мIT Press, pp. 119-150.

Ulrich, Karl T., y Eppinger, Steven D. (2009), Diseño y desarrollo de productos, México, Mc Graw Hill.

USPTO (s.f.), "Environmentally Sound Technologies (ETs) Concordance", en United States Patent and Trademark Office. Consultada el 27 de noviembre de 2012 en http://www.uspto.gov/web/patents/classification/international/est_concordance. htm

Watts, Duncan J. (1999), Small Worlds: The Dynamics of Network between Order and Randomness, Pincenton, Princenton University Press. 